\title{
Novel Inflammatory Biomarkers as Additional Value to Traditional Risk-factors in Cardiovascular Risk Stratification
}

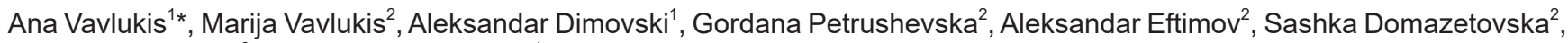 \\ Almasa Demirovikj ${ }^{3}$, Kristina Mladenovska ${ }^{1}$ \\ ${ }^{1}$ Faculty of Pharmacy, Ss. Cyril and Methodius University, Skopje, Republic of Macedonia; ${ }^{2}$ Faculty of Medicine, Ss. Cyril and \\ Methodius University, Skopje, Republic of Macedonia; ${ }^{3}$ PZU Ena-Medikal, Skopje, Republic of Macedonia
}

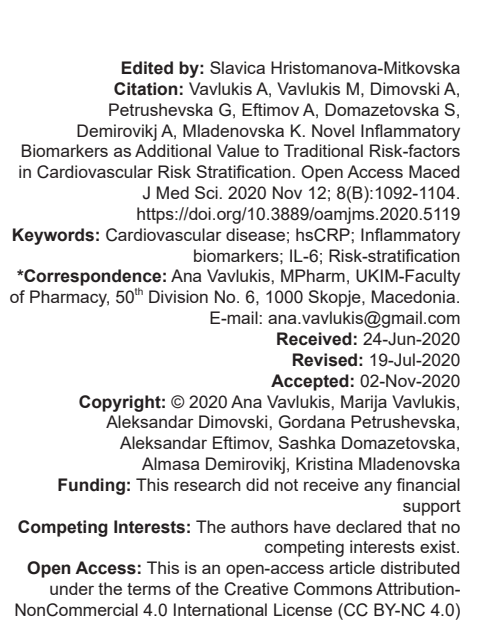

Abstract

BACKGROUND:: There is a growing interest in the possible role of inflammatory biomarkers, such as interleukins chemokines, growth factors, and acute-phase proteins, in cardiovascular risk-stratification.

AIM: The aim of the study was to determine a possible correlation between the subjects' cardiovascular risk profile and various inflammatory markers and to assess the sole use of IL-6 in CVD risk prediction.

MATERIALS AND METHODS: Seventy-five healthy subjects participated. EUROSCORE, lipid, glycemic, and inflammatory markers were analyzed. Chi-square test, t-test, one-way ANOVA, Mann-Whitney, and Kruskal-Wallis tests were used. Significance was determined at $<0.05$

RESULTS: A multivariate analysis revealed 12 markers to be independently associated with CVD risk - LDL-C, TG ApoB, HbA1c, hsCRP, IL-6, and IL-1A as markers of higher, and HDL-C, IL-4, II-10, VEGF, and EGF as markers of lower CVD risk. IL-6 levels $>1 \mathrm{pg} / \mathrm{ml}$ were positively correlated with female gender, age $>55$ years, EUROSCORE $\geq 3$, risk age, SBP, hsCRP $>2 \mathrm{mg} / \mathrm{L}$, and IL-2 ( $p=0.025, p=0.013, p=0.025, p=0.011, p=0.026, p=0.046$, and $p=0.018$ ). Except for total CVD risk and risk age, the same variables were identified to be independently associated with IL-6.

CONCLUSION: Inflammatory biomarkers, especially hSCRP and IL-6, have a statistically significant, added predictive power in cardiovascular risk stratification.

\section{Introduction}

Cardiovascular disease (CVD) remains a leading cause of morbidity and mortality, despite improvements in outcomes. Although age-adjusted coronary artery disease mortality has declined since the 1980s, inequalities between countries persist and many risk factors, particularly obesity and diabetes mellitus (DM), have been increasing substantially [1].

Apart from the conventional major cardiovascular risk factors included in the currently used risk charts, there are other risk factors that could be relevant for assessing total CVD risk [2]. The previous has led to a vast investigation of CVD risk-modifiers, such as N-terminal fragment of prohormone B-type natriuretic peptide levels, von Willebrand factor antigen levels, fibrinogen levels, chronic kidney disease, leukocyte count, C-reactive protein (CRP) levels, homocysteine levels, uric acid levels, coronary artery calcium scores, carotid intimamedia thickness, peripheral arterial disease, and pulse wave velocity [3]. The nontraditional risk factors have resulted in varying degrees of improvement in discrimination and reclassification of risk, including no improvement [4].

Advances in vascular biology have established the interaction of the innate immune system and atherosclerosis, with emerging clinical studies linking chronic inflammation to future CV events [5]. Several biomarkers have been addressed to improve the identification of at-risk asymptomatic patients regarding the central role of inflammation in atherosclerosis initiation and progression [6].

The MRFIT (Multiple Risk Factor Intervention Trial) was one of the first prospective epidemiology studies to show a strong relationship between levels of high sensitivity CRP (hsCRP) and mortality from coronary heart disease in high-risk middle-aged men, as well as an association between increasing hsCRP levels and subsequent myocardial infarction and/or stroke rate in apparently healthy men [7]. The WHS (Women's Health Study) showed hsCRP as a stronger predictor of CV events compared to lowdensity lipoprotein cholesterol (LDL-C) in over 27000 apparently healthy women [8]. 
The Emerging Risk Factor Collaboration (ERFC), while reviewing a possible association among hsCRP levels, CV risk factors, and vascular risk in 160 309 individuals, found a similar magnitude-increased risk of higher hsCRP levels regarding coronary heart disease (RR: 1.68; 95\% Cl: 1.59-1.78), ischemic stroke (RR: $1.46 ; 95 \% \mathrm{Cl}: 1.32-1.61$ ), and death from vascular causes (RR: $1.82 ; 95 \% \mathrm{Cl}$ : 1.66-2.00), all risk ratios similar to those seen for hyperlipidemia [9].

Despite the vast clinical studies addressing the role of CRP in CVD settings, the Jupiter (Justification for the Use of Statins in Prevention: An Intervention Trial Evaluating Rosuvastatin) trial is said to be the first to postulate hsCRP's potential role in CVD prevention. Randomizing 17,802 middleaged to elderly, low to intermediate-risk patients with LDL-C $<130 \mathrm{mg} / \mathrm{dl}$ and $\mathrm{hsCRP}>2 \mathrm{mg} / \mathrm{l}$ to rosuvastatin $20 \mathrm{mg}$ versus placebo, the trial reported a robust $44 \%$ RRR (95\% Cl: $31 \%$ to $54 \%$; p < 0.00001) in the primary endpoint of myocardial infarction, stroke, revascularization, hospitalization for unstable angina, or death; with $50 \%$ and $37 \%$ reductions in LDL-C and hsCRP levels in the rosuvastatin arm, respectively. Showing lowest $\mathrm{CV}$ events incidence in subjects achieving both low LDL-C and low hsCRP levels, the Jupiter study findings are used as evidence-based justification regarding the use of hsCRP as a screening tool for CVD risk stratification and as a marker in the treatment management process [10], [11].

While hsCRP is a clinically proven risk assessment tool, the biomarker itself is unlikely to provide an effective target for intervention. The previous has led to an increased interest in the inflammatory background of CVD as a whole [12]. The disturbance of the oxidative/anti-oxidative homeostasis in CVD perpetuates an inflammatory response in the subendothelial space, activating various immune cells to secrete pro-inflammatory molecules such as tumor necrosis factor- $\alpha$ (TNF- $\alpha)$ and interleukin-1 (IL-1). These promote secretion of monocyte chemoattractant protein-1 (MCP-1), which leads to monocyte recruitment and macrophage activation [13]. The process of atherosclerosis itself leads to the secretion of additional cytokines such as IL-10 and IL-6 and increases hepatic CRP synthesis, resulting in phagocytosis in the atherosclerotic plaque [14]. In addition, MCP-1 is thought to be one of the major regulators of vascular endothelial growth factor (VEGF) and epidermal growth factor (EGF), both cytoprotective and proangiogenic growth factors [15].

Upstream movement in the inflammatory cascade from CRP to IL-6-IL-1 has recently provided novel therapeutic opportunities for atheroprotection. Preliminary data from a single-dose study of tocilizumab (a humanized anti-IL-6 receptor antibody) in non-ST elevation myocardial infarction showed a reduction in the area under the CRP curve (ClinicalTrial. gov NCT01491074) [16]. CANTOS (Canakinumab
Anti-inflammatory Thrombosis Outcomes Study), with a population of more than 10,000 patients, is a study set out to test whether blocking the pro-inflammatory cytokine interleukin-1ß (IL-1ß) with canakinumab (a fully human monoclonal anti-IL-1 $\beta$ antibody), in comparison to placebo, reduces the rate of recurrent myocardial infarction, stroke, and cardiovascular death among patients who remain at high risk due to persistent CRP elevation ( $\geq 2 \mathrm{mg} / \mathrm{l})$. Canakinumab directly inhibits the IL-1 $\beta-I L-6$ to CRP axis, with no effect on LDL-C; thus, CANTOS will be the first large scale test of the inflammation hypothesis of atherothrombosis [17].

Although cytokine-targeted therapy with biological agents is slowly taking the focus regarding the anti-inflammatory CVD hypothesis, statins, the golden standard for cholesterol-lowering in patients at risk of/with CVD, have numerous anti-inflammatory and immunomodulatory pleiotropic properties yet to be determined and translated into the clinical practice.

The results that will be presented in the following paper are part of a research project evaluating the traditional versus pleiotropic actions of rosuvastatin in apparently healthy subjects at medium-to-high CVD risk.

The aim of the study was to determine a possible correlation between the subjects' cardiovascular risk profile and various inflammatory markers; to assess whether IL-6 can be a better inflammatory marker than hsCRP in CVD risk prediction.

\section{Materials and Methods}

\section{Study design, subjects, and protocol}

This is a cross-sectional cohort study of subjects with moderate to high $\mathrm{CV}$ risk without known cardiovascular disease. Seventy-five adult ambulatory patients (aged 50.5 \pm 12 , 1 year, 41 women, 34 men) from the University Clinic of Cardiology in Skopje were included in the study. The study protocol was approved by the Clinical Studies and Ethics Committee of UKIM-Faculty of Medicine, Skopje. Every subject received verbal and written information and gave written consent before the start of the study. The study analyzed demographic indicators, family history, traditional CVD risk factors (smoking, arterial hypertension [HTA], hyper/dyslipidemia [HLP], diabetes mellitus [DM], and obesity), calculated EUROSCORE risk, SCORE risk age, SCORE relative risk, using a predefined algorithm: www.heartscore. org, and determined lipoprotein profile indicators (total cholesterol [Chol], low-density lipoprotein cholesterol [LDL-C], high-density lipoprotein cholesterol [HDL-C], triglycerides [TG], apolipoprotein A1 [ApoA1], apolipoprotein B [ApoB], and lipoprotein 
[a] $L p(a)$ ), glycemic profile indicators (fasting glucose, glycosylated hemoglobin $\mathrm{A} 1 \mathrm{c}[\mathrm{HbA} 1 \mathrm{c}])$, and inflammatory profile indicators (interleukins [IL-1A, IL-1B, IL-2, IL-4, IL-6, IL-8 and IL-10]), cytokines (tumor necrosis factor- $\alpha[$ TNF- $\alpha]$ and interferon- $\gamma$ [IFN- $\gamma]$ ), chemokines (monocyte chemoattractant protein-1 [MCP-1]), growth factors (epidermal growth factor [EGF] and vascular endothelial growth factor [VEGF]), and acute phase proteins (C-reactive protein [CRP]).

Data were obtained from the patients' medical history, physical examination, and blood samples taken on the day of the clinical visit. A comparative analysis was performed based on the presence/absence of: HLP, DM, and a hsCRP value $>2 \mathrm{mg} / \mathrm{L}$.

\section{Methods}

$4.0 \mathrm{~mL}$ venous blood in two EDTA/K3 vacuum tubes was taken from each patient and immediately transported (by a chain maintaining a $4^{\circ} \mathrm{C}$ temperature of the samples) and analyzed after sampling. Biochemical parameters were determined at the University Clinic of Clinical Biochemistry, Skopje, and the inflammatory markers were determined at the Institute of Pathology, UKIM-Faculty of Medicine, Skopje, with the methods described in Appendix 1.

\section{Statistical analysis}

Table 1: General characteristics of the study population

\begin{tabular}{|c|c|c|c|c|c|c|c|c|c|c|}
\hline Variable & No (\%) & $\begin{array}{l}\text { WITH HLP } \\
(\%)\end{array}$ & $\begin{array}{l}\text { WITHOUT } \\
\text { HLP }\end{array}$ & Sig & $\begin{array}{l}\text { WITH } \\
\text { DM }\end{array}$ & $\begin{array}{l}\text { WITHOUT } \\
\text { DM }\end{array}$ & Sig & $\mathrm{hsCRP}>2$ & $\mathrm{hsCRP}<2$ & Sig \\
\hline Gender & $75(100 \%)$ & $64(85.3 \%)$ & $11(14.7 \%)$ & ns & $15(20 \%)$ & $60(80 \%)$ & Ns & $38(50.7 \%)$ & $37(49.3 \%)$ & 0.014 \\
\hline Female & $41(54.7 \%)$ & $36(48 \%)$ & $5(6.7 \%)$ & & $10(13.3 \%)$ & $31(41.3 \%)$ & ORf 1.4 & $26(34.7 \%)$ & $15(20.0 \%)$ & ORf 1.9 \\
\hline Male & $34(45.3 \%)$ & $28(37.3 \%)$ & $6(8 \%)$ & & $5(6.7 \%)$ & $29(38.7 \%)$ & $(\mathrm{Cl} 0.7-3.1)$ & $12(16.0 \%)$ & $22(29.3 \%)$ & (CI 1.1-3.2) \\
\hline Age & $50.5 \pm 12.1$ & $51.1 \pm 11.2$ & $47.2 \pm 16.7$ & ns & $59.7 \pm 9.7$ & $48.2 \pm 11.6$ & 0.001 & $51.4 \pm 12.4$ & $49.5 \pm 11.9$ & ns \\
\hline Female & $52.5 \pm 10.8$ & & & & & & & & & \\
\hline Male & $48.1 \pm 13.3$ & & & & & & & & & \\
\hline Smoking & $14(18 . .7)$ & $13(20.3 \%)$ & $1(9.1 \%)$ & ns & $5(33.3 \%)$ * & $9(15.0 \%)$ * & ns & $5(13.2 \%)$ & $9(24.3 \%)$ & Ns \\
\hline Family history & $10(13.3)$ & $9(14.1 \%)$ & $1(9.1 \%)$ & ns & 0 & $10(16.7 \%)$ * & ns & $5(13.2 \%)$ & $5(13.5 \%)$ & Ns \\
\hline DM & $15(20)$ & $14(21.9 \%)$ & $1(9.1 \%)$ & ns & & & & $8(21.1 \%)$ & $7(18.9 \%)$ & ns \\
\hline Pre-diabetes & 35 (46.7) & $28(43.8 \%)$ & $7(63.6 \%)$ & ns & 1 & $35(58.3 \%)$ & 0.000 & $18(47.4 \%)$ & $17(45.9 \%)$ & ns \\
\hline HLP & & & & & $14(93.3 \%)^{*}$ & $50(83.3 \%)$ * & ns & $33(86.8 \%)$ & $31(83.8 \%)$ & ns \\
\hline $\mathrm{BH}(\mathrm{cm})$ & $169.0 \pm 9.7$ & $169 \pm 9.3$ & $169.2 \pm 12.7$ & ns & $168.7 \pm 10.5$ & $169.7 \pm 9.7$ & ns & $167.3 \pm 10.3$ & $170.8 \pm 8.9$ & ns \\
\hline $\mathrm{BW}(\mathrm{kg})$ & $79.1 \pm 14.0$ & $79.3 \pm 14.5$ & $77.8 \pm 12.1$ & ns & $78.1 \pm 15.4$ & $79.2 \pm 13.9$ & ns & $79.4 \pm 12.7$ & $78.7 \pm 15.6$ & ns \\
\hline BMI & $27.6 \pm 4.1$ & $27.7 \pm 4.2$ & $27.2 \pm 3.3$ & ns & $27.6 \pm 5.1$ & $27.6 \pm 3.9$ & ns & $28.3 \pm 3.5$ & $26.9 \pm 4.6$ & ns \\
\hline $\begin{array}{l}\text { SBP } \mathrm{mmHg} \\
\text { EUROSCORE risk profile }\end{array}$ & \multicolumn{10}{|c|}{ EUROSCORE risk profile } \\
\hline $\begin{array}{l}\text { EUROSCORE (\%) } \\
\text { Low risk }\end{array}$ & $\begin{array}{l}3.5 \pm 4.3 \\
12(16 \%)\end{array}$ & $\begin{array}{l}3.8 \pm 4.5 \\
9(14.1 \%)\end{array}$ & $\begin{array}{l}1.4 \pm 1.3 \\
3(27.3 \%)\end{array}$ & 0.078 & $\begin{array}{l}6.7 \pm 6.0 \\
0\end{array}$ & $\begin{array}{l}2.7 \pm 3.3 \\
12(20 \%)\end{array}$ & 0.001 & $\begin{array}{l}3.8 \pm 3.8 \\
6(15.8 \%)\end{array}$ & $\begin{array}{l}4.1 \pm 5.5 \\
6(16.2 \%)\end{array}$ & ns \\
\hline High risk & $13(17.3 \%)$ & $13(20.3 \%)$ & 0 & $0.073^{* *}$ & $4(26.7 \%)$ & $9(15 \%)$ & & $8(21.1 \%)$ & $5(13.5 \%)$ & \\
\hline Very high risk & $7(9.3 \%)$ & $7(10.9 \%)$ & 0 & & $4(26.7 \%)$ & $3(5 \%)$ & & $4(10.5 \%)$ & $3(8.1 \%)$ & \\
\hline Risk age & $56.2 \pm 7.9$ & $56.5 \pm 7.7$ & $54.7 \pm 9.1$ & ns & $60.7 \pm 4.6$ & $55.1 \pm 8.2$ & 0.014 & $55.9 \pm 9.8$ & $55.3 \pm 8.1$ & ns \\
\hline Relative risk & $2.7 \pm 1.8$ & $3.0 \pm 2.0$ & $2.0 \pm 0.8$ & 0.006 & $3.9 \pm 2.9$ & $2.5 \pm 1.4$ & 0.008 & $2.9 \pm 1.5$ & $3.1 \pm 2.7$ & ns \\
\hline No of pts. with risk age & $47(62.7 \%)$ & $40(62.5 \%)$ & $7(63.6 \%)$ & ns & $6(40 \%)$ & $41(68.3 \%)$ & $0.043^{\star *}$ & $22(57.9 \%)$ & $25(67.6 \%)$ & ns \\
\hline >biological age & & & & & & & $\begin{array}{l}\text { ORnonDM2.5 } \\
\text { (Cl 1.0-6.3) }\end{array}$ & & & \\
\hline \multicolumn{11}{|l|}{ LP profile } \\
\hline Chol $>5 \mathrm{mmol} / \mathrm{L}$ & $65(86.7 \%)$ & $54(91.1 \%)$ & $11(14.7 \%)$ & 0.000 & $14(93.3 \%)$ & $50(83.3 \%)$ & ns & $21(46.7 \%)$ & $20(44.4 \%)$ & ns \\
\hline Chol (mmol/L) & $6.3 \pm 1.4$ & $6.7 \pm 1.1$ & $4.2 \pm 0.3$ & 0.000 & $6.5 \pm 1.4$ & $6.3 \pm 1.4$ & ns & $6.8 \pm 1.3$ & $6.3 \pm 1.5$ & ns \\
\hline LDL-C (mmol/L) & $4.0 \pm 1.4$ & $4.3 \pm 1.2$ & $2.2 \pm 0.4$ & 0.001 & $4.2 \pm 1.4$ & $3.9 \pm 1.4$ & ns & $4.3 \pm 1.4$ & $3.8 \pm 1.5$ & ns \\
\hline $\mathrm{HDL}-\mathrm{C}(\mathrm{mmol} / \mathrm{L})$ & $1.4 \pm 0.3$ & $1.4 \pm 0.4$ & $1.2 \pm 0.2$ & ns & $1.4 \pm 0.4$ & $1.4 \pm 0.3$ & ns & $1.5 \pm 0.4$ & $1.3 \pm 0.3$ & 0.066 \\
\hline TG $(\mathrm{mmol} / \mathrm{L})$ & $2.3 \pm 2.0$ & $2.4 \pm 2.1$ & $1.5 \pm 0.9$ & ns & $3.3 \pm 3.9$ & $1.9 \pm 1.1$ & 0.018 & $2.0 \pm 1.1$ & $2.8 \pm 3.2$ & ns \\
\hline ApoA1 (g/L) & $1.6 \pm 0.3$ & $1.7 \pm 0.3$ & $1.5 \pm 0.2$ & ns & $1.6 \pm 0.3$ & $1.6 \pm 0.3$ & ns & $1.7 \pm 0.4$ & $1.6 \pm 0.3$ & ns \\
\hline ApoB $(g / L)$ & $1.4 \pm 0.4$ & $1.5 \pm 0.3$ & $1.0 \pm 0.4$ & 0.000 & $1.4 \pm 0.4$ & $1.4 \pm 0.4$ & ns & $1.5 \pm 0.4$ & $1.4 \pm 0.4$ & ns \\
\hline Lp(a) (mg/tdl) & $42.1 \pm 58.8$ & $39.7 \pm 55.5$ & $55.8 \pm 77.3$ & ns & $27.5 \pm 20.3$ & $45.7 \pm 64.6$ & ns & $40.7 \pm 43.4$ & $39.8 \pm 50.9$ & ns \\
\hline
\end{tabular}

Continuous variables are expressed as mean value $\pm S D$ and categorical variables are expressed as absolute numbers. Chi-square test, t-test, and oneway ANOVA were used for the variables that follow the normal distribution, while nonparametric tests, such as Mann-Whitney and Kruskal-Wallis tests, were used to analyze the continuous variables that deviated from the normal distribution. Correlations and ROC curves were computed, and uni- and multivariate logistic regression analysis was made. Results were considered statistically significant when $p \leq 0.05$. Data were analyzed using the IBM SPSS 19.0 statistical software.

\section{Results}

\section{population}

\section{General characteristics of the study}

We included 75 patients with moderate to high $\mathrm{CV}$ risk in the study. General characteristics of the study population in a comparative manner (as a function of the presence of HLP, diabetes, or hsCRP >2 $\mathrm{mg} / \mathrm{L}$ ) are shown in Table 1.

The total estimated EUROSCORE (\%) in the study population is 3.5 , which places it in the group of individuals with a moderate-to-high 10-year risk of fatal CVD. The calculated relative risk is 2,7 , or nearly a 3 -fold 
Table 1: (Continued)

\begin{tabular}{|c|c|c|c|c|c|c|c|c|c|c|}
\hline Variable & No $(\%)$ & $\begin{array}{l}\text { WITH HLP } \\
(\%)\end{array}$ & $\begin{array}{l}\text { WITHOUT } \\
\text { HLP }\end{array}$ & Sig & $\begin{array}{l}\text { WITH } \\
\text { DM }\end{array}$ & $\begin{array}{l}\text { WITHOUT } \\
\text { DM }\end{array}$ & Sig & hsCRP $>2$ & hsCRP<2 & Sig \\
\hline \multicolumn{11}{|l|}{ Glycemic profile } \\
\hline Glycemia & $6.4 \pm 4.9$ & $6.6 \pm 5.2$ & $5.7 \pm 2.1$ & $\mathrm{~ns}^{* *}$ & $8.9 \pm 4.5$ & $5.8 \pm 4.8$ & 0.025 & $5.8 \pm 1.8$ & $7.1 \pm 6.7$ & ns \\
\hline $\begin{array}{l}\text { Glycated hemoglobin } \\
\text { (HbA1c-\%) }\end{array}$ & $6.0 \pm 1.0$ & $6.0 \pm 1.0$ & $5.7 \pm 0.4$ & $\mathrm{~ns}^{* *}$ & $7.2 \pm 1.5$ & $5.7 \pm 0.3$ & 0.000 & $5.9 \pm 0.6$ & $6.1 \pm 1.2$ & ns \\
\hline \multicolumn{11}{|l|}{$\begin{array}{l}\text { (HbA1c-\%) } \\
\text { HbA1c categorical }\end{array}$} \\
\hline$<5.6 \mathrm{mmol} / \mathrm{L}$ & $24(32 \%)$ & $21(32.8 \%)$ & $3(27.3)$ & & 0 & $24(40 \%)$ & & $12(31.6 \%)$ & $12(32.4 \%)$ & \\
\hline $5.6-6.5 \mathrm{mmol} / \mathrm{L}$ & $41(54.7 \%)$ & $34(53.1 \%)$ & $7(63.6 \%)$ & $\mathrm{ns}^{* *}$ & $5(33.3 \%)$ & $36(60 \%)$ & 0.000 & $21(55.3 \%)$ & $20(54.1 \%)$ & ns \\
\hline$>6.5 \mathrm{mmol} / \mathrm{L}$ & $10(13.3 \%)$ & $9(14.1 \%)$ & $1(9.1 \%)$ & & $10(66.7 \%)$ & 0 & & $5(13.2 \%)$ & $5(13.5 \%)$ & \\
\hline Uric acid & $314.6 \pm 73.6$ & $313.2 \pm 74.6$ & $318.1 \pm 72.4$ & $\mathrm{~ns}^{* *}$ & $305.9 \pm 71.7$ & $316.7 \pm 74.4$ & ns & $317.6 \pm 68.9$ & $311.4 \pm 78.8$ & \\
\hline \multicolumn{11}{|c|}{ Biomarkers of inflammation/proliferation } \\
\hline $\mathrm{hsCRP}(\mathrm{mg} / \mathrm{L})$ & $3.2 \pm 3.8$ & $3.2 \pm 3.8$ & $3.0 \pm 3.9$ & $\mathrm{~ns}^{* *}$ & $3.4 \pm 3.4$ & $3.1 \pm 3.9$ & $\mathrm{~ns}^{* *}$ & $5.2 \pm 4.5$ & $1.1 \pm 0.3$ & 0.000 \\
\hline NO of pts. with hsCRP & $38(50.7 \%)$ & $33(51.6 \%)$ & $5(45.5 \%)$ & $\mathrm{ns}^{* *}$ & $8(53.3 \%)$ & $30(50 \%)$ & $\mathrm{ns}^{* *}$ & $38(50.7 \%)$ & $37(49.3 \%)$ & $\mathrm{ns}^{* *}$ \\
\hline \multicolumn{11}{|l|}{$>2 \mathrm{mg} / \mathrm{L}$} \\
\hline $\mathrm{hsCRP} \leq 1 \mathrm{mg} / \mathrm{L}$ & $21(28 \%)$ & $18(28.1 \%)$ & $4(36.4 \%)$ & & $4(26.7 \%)$ & $18(30 \%)$ & & $22(29.5)$ & & \\
\hline hsCRP $>1 \leq 3 \mathrm{mg} / \mathrm{L}$ & $30(40 \%)$ & $25(39.1 \%)$ & $4(36.4 \%)$ & $\mathrm{ns}^{* *}$ & $5(33.3 \%)$ & $24(40 \%)$ & $\mathrm{ns}^{\star *}$ & $29(38.7 \%)$ & & $\mathrm{ns}^{\star *}$ \\
\hline $\mathrm{hsCRP}>3 \mathrm{mg} / \mathrm{L}$ & $24(32 \%)$ & $21(32.8 \%)$ & $3(27.3 \%)$ & & $6(40 \%)$ & $18(30 \%)$ & & $24(32 \%)$ & & \\
\hline IL-2 & $1.8 \pm 1.0$ & $1.8 \pm 0.9$ & $2.0 \pm 1.3$ & $\mathrm{~ns}^{* *}$ & $1.8 \pm 1.1$ & $1.8 \pm 1.0$ & $\mathrm{~ns}^{* *}$ & $1.7 \pm 0.8$ & $2.0 \pm 1.1$ & $\mathrm{~ns}^{* *}$ \\
\hline IL-4 & $2.0 \pm 0.7$ & $2.0 \pm 0.7$ & $1.9 \pm 0.7$ & $\mathrm{~ns}^{* *}$ & $1.9 \pm 0.8$ & $2.0 \pm 0.7$ & $\mathrm{~ns}^{* *}$ & $1.9 \pm 0.6$ & $2.0 \pm 0.8$ & $\mathrm{~ns}^{* *}$ \\
\hline \multirow[t]{2}{*}{ IL-6 } & $1.4 \pm 1.6$ & $1.4 \pm 1.8$ & $1.1 \pm 0.7$ & $\mathrm{~ns}^{* *}$ & $1.3 \pm 0.8$ & $1.4 \pm 1.8$ & $\mathrm{~ns}^{* *}$ & $1.9 \pm 2.2$ & $1.0 \pm 0.6$ & $0.016^{*}$ \\
\hline & $4.5 \pm 5.6$ & $4.3 \pm 4.8$ & $5.6 \pm 9.4$ & $\mathrm{~ns}^{* *}$ & $2.7 \pm 2.0$ & $4.8 \pm 6.0$ & $\mathrm{~ns}^{* *}$ & $4.6 \pm 5.5$ & $4.3 \pm 5.8$ & $\begin{array}{l}0.017^{* *} \\
\mathrm{~ns}^{* *}\end{array}$ \\
\hline IL-10 & $0.6 \pm 0.5$ & $0.6 \pm 0.5$ & $0.5 \pm 0.3$ & $\mathrm{~ns}^{* *}$ & $0.5 \pm 0.3$ & $0.6 \pm 0.5$ & $\mathrm{~ns}^{* *}$ & $0.6 \pm 0.3$ & $0.6 \pm 0.6$ & $\mathrm{~ns}^{* *}$ \\
\hline VEGF & $35.3 \pm 31.8$ & $37.3 \pm 32.3$ & $23.3 \pm 26.6$ & $\mathrm{~ns}^{* *}$ & $33.9 \pm 33.7$ & $35.6 \pm 31.7$ & $\mathrm{~ns}^{* *}$ & $40.7 \pm 34.4$ & $30.1 \pm 28.7$ & $\mathrm{~ns}^{* *}$ \\
\hline IFN-y & $0.3 \pm 0.8$ & $0.4 \pm 0.9$ & $0.2 \pm 0.1$ & $\mathrm{~ns}^{* *}$ & $0.1 \pm 0.1$ & $0.4 \pm 0.9$ & $\mathrm{~ns}^{* *}$ & $0.4 \pm 1.0$ & $0.3 \pm 0.6$ & $\mathrm{~ns}^{\star *}$ \\
\hline TNF- $\alpha$ & $2.0 \pm 1.8$ & $2.1 \pm 1.8$ & $1.8 \pm 1.3$ & $\mathrm{~ns}^{* *}$ & $1.5 \pm 0.7$ & $2.2 \pm 1.9$ & $\mathrm{~ns}^{* *}$ & $2.0 \pm 1.1$ & $2.1 \pm 2.2$ & $\mathrm{~ns}^{* *}$ \\
\hline IL-1A & $0.3 \pm 0.2$ & $0.3 \pm 0.1$ & $0.3 \pm 0.2$ & $n \mathrm{n}^{* *}$ & $0.2 \pm 0.1$ & $0.3 \pm 0.2$ & $\mathrm{~ns}^{* *}$ & $0.3 \pm 0.1$ & $0.3 \pm 0.2$ & $\mathrm{~ns}^{* *}$ \\
\hline $\mathrm{IL}-1 \mathrm{~B}$ & $1.6 \pm 1.0$ & $1.6 \pm 1.1$ & $1.4 \pm 0.9$ & $\mathrm{~ns}^{* *}$ & $1.2 \pm 0.6$ & $1.6 \pm 1.1$ & $\mathrm{~ns}^{* *}$ & $1.6 \pm 0.9$ & $1.6 \pm 1.2$ & $\mathrm{~ns}^{\star *}$ \\
\hline \multirow[t]{2}{*}{ MCP-1 } & $83.4 \pm 73.9$ & $85.4 \pm 76.7$ & $70.9 \pm 55.5$ & $\mathrm{~ns}^{* *}$ & $130.2 \pm$ & $73.9 \pm 63.6$ & $0.015^{*}$ & $89.9 \pm 75.6$ & $77.4 \pm 72.9$ & $\mathrm{~ns}^{\star *}$ \\
\hline & & & & & 102.8 & & $0.050^{* *}$ & & & \\
\hline EGF & $46.7 \pm 63.1$ & $48.4 \pm 64.5$ & $36.6 \pm 55.3$ & $n \mathrm{~ns}^{* *}$ & $49.4 \pm 58.4$ & $46.1 \pm 64.5$ & $\mathrm{~ns}^{* *}$ & $54.1 \pm 70.0$ & $39.9 \pm 56.1$ & $\mathrm{~ns}^{* *}$ \\
\hline
\end{tabular}

Table 2: Cytokine concentration ranges in the "healthy adult population" reported by different authors and in our study group

\begin{tabular}{|c|c|c|c|c|}
\hline Cytokine (pg/ml) & Mean $^{1}$ (range) & $\operatorname{Mean}^{2}$ (range) & $\operatorname{Mean}^{3}$ (range) & Study group \\
\hline $\mathrm{IL} 2$ & $1.1(1.1-9.2)$ & $14(9.4-15.9)$ & $6.46(0.03-90)$ & $1.8 \pm 1.0(0.0-5.55)$ \\
\hline IL-4 & $2.7(1.9-3.8)$ & & $0.10(0.01-3.0)$ & $2.0 \pm 0.7(0.0-3.99)$ \\
\hline IL-6 & $4.6(1.1-10.8)$ & & $0.73(0.02-9.0)$ & $1.4 \pm 1.6(0.12-11.29)$ \\
\hline IL-8 & $3.9(1.0-8.2)$ & $29.3(24.4-35.9)$ & $7.21(0.08-116)$ & $4.5 \pm 5.6(0.41-29.45)$ \\
\hline IL-1A & & 1.4 (LLOD)* & $0.12(0.40-1.40)$ & $0.3 \pm 0.2(0.0-0.87)$ \\
\hline IL-1B & $2.6(0.8-3.9)$ & 3.2 (LLOD) & $0.01(0.02-0.70)$ & $1.6 \pm 1.0(0.0-5.35)$ \\
\hline IFN-y & $77.1(48.4-127.6)$ & & $13.43(7-124)$ & $0.3 \pm 0.8(0.0-6.07)$ \\
\hline TNF- $\alpha$ & $35.3(14.2-61.7)$ & & $5.92(0.10-98.0)$ & $2.0 \pm 1.8(0.38-11.63)$ \\
\hline VEGF & $11(4.6-20.3)$ & $61.6(32-118.9)$ & $0.43(0.01-9.0)$ & $35.3 \pm 31.8(5.05-147.76)$ \\
\hline MCP-1 & $16(10.6-24.0)$ & $41.5(20.1-78.9)$ & $18.24(2.0-48.0)$ & $83.4 \pm 73.9(1.26-314.66)$ \\
\hline
\end{tabular}

increased CVD risk compared to an equivalent population with normal risk factor values. The risk age of the study population (a person of the same sex and risk level, but with ideal risk factor values) is $\sim 56$ years, 6 years higher than the actual average age. More than half of the study population $(62.7 \%)$ has a risk age $>$ biological age.

The presence of HLP shows a significant correlation with a relative risk $(3.0 \pm 2.0$ with $H L P$, compared to $2.0 \pm 0.8$ without HLP, $p=0.006$ ).

The presence of DM is significantly correlated with HTA (14 [93.3\%] vs. 40 [66.7\%], p = 0.034; OR 1.3 [Cl 1.1-1.5]), total estimated EUROSCORE (\%) (6.7 \pm 6.0 vs. $2.7 \pm 3.3, p=0.001)$, risk age $(60.7 \pm 4.6$ vs. $55.1 \pm 8.2, p=0.014)$, and relative risk $(3.9 \pm 2.9$ vs. 2.5 $\pm 1.4, p=0.008)$, with/without $D M$, respectively. $D M$ is positively correlated with MCP-1 levels (130.2 \pm 102.8 vs. $73.9 \pm 63.6$ in the group without $\mathrm{DM}, \mathrm{p}=0.050)$.

hsCRP levels > $2 \mathrm{mg} / \mathrm{L}$ are significantly correlated with female gender (26 (34.7\%) vs. $15(20.0 \%)$ in the group with hsCRP levels < $2 \mathrm{mg} / \mathrm{L}, \mathrm{p}=0.014$; ORf 1,9 (Cl 1.1-3.2), and HTA [33(86.8\%) vs. 21(56.8\%) in the group with hsCRP levels < 2 mg/L, $p=0.004$; OR $1.9(\mathrm{Cl}$
1.3-2.9)]. hsCRP levels $>2 \mathrm{mg} / \mathrm{L}$ are positively correlated with IL-6 levels $(1.9 \pm 2.2$ vs. $1.0 \pm 0.6, p=0.017)$.

\section{Inflammatory biomarkers}

At the present moment, there are no defined ranges of "normal values" of inflammatory biomarkers. Table 2 shows cytokine concentration (mean and range) measured in our study cohort and ranges measured by different investigators in the healthy adult population, with the intention simply to give you an overview of the current situation of reported ranges of inflammatory biomarkers.

We performed a gender analysis of inflammatory biomarkers. Compared to males, females have higher levels of hsCRP and IL-6 (4.2 \pm 4.7 vs. $1.9 \pm$ 1.5 and $1.9 \pm 2.1$ vs. $0.9 \pm 0.5$, respectively), statistically significant with both parametric and nonparametric tests $(p=0.008 / p=0.002$ and $p=0.010 / p=0.003$, respectively) (Table 3 ).

We analyzed possible interrelations between the inflammatory biomarkers, to identify significant correlations. We revealed numerous significant 
Table 3: Gender distribution of inflammatory biomarker levels

\begin{tabular}{|c|c|c|c|c|}
\hline Parameter & Female 0/Male 1 & $\mathrm{~N}$ & Mean \pm SD & $\begin{array}{l}\text { Sig (parametric) } \\
\text { Sig* (Mann-Whitney U test) }^{*}\end{array}$ \\
\hline \multirow[t]{2}{*}{ CRP } & 0 (f) 41 & 41 & $4.2 \pm 4.7$ & 0.008 \\
\hline & 1 (m) 34 & 34 & $1.9 \pm 1.5$ & $0.002^{*}$ \\
\hline \multirow[t]{2}{*}{ IL-2 } & 0 & 39 & $1.9 \pm 1.1$ & ns \\
\hline & 1 & 32 & $1.8 \pm 0.8$ & \\
\hline \multirow[t]{2}{*}{ IL-4 } & 0 & 39 & $2.0 \pm 0.6$ & ns \\
\hline & 1 & 32 & $1.9 \pm 0.8$ & \\
\hline \multirow[t]{2}{*}{ IL-6 } & 0 & 39 & $1.9 \pm 2.1$ & $0.010 / 0.003^{*}$ \\
\hline & 1 & 32 & $0.9 \pm 0.5$ & \\
\hline \multirow[t]{2}{*}{ IL-8 } & 0 & 39 & $3.6 \pm 3.8$ & ns \\
\hline & 1 & 32 & $5.5 \pm 7.2$ & \\
\hline \multirow[t]{2}{*}{ IL-10 } & 0 & 39 & $0.6 \pm 0.6$ & ns \\
\hline & 1 & 32 & $0.6 \pm 0.3$ & \\
\hline \multirow[t]{2}{*}{ IL-1A } & 0 & 39 & $0.2 \pm 0.1$ & ns \\
\hline & 1 & 32 & $0.3 \pm 0.2$ & \\
\hline \multirow[t]{2}{*}{ IL-1B } & 0 & 39 & $1.4 \pm 0.8$ & ns \\
\hline & 1 & 32 & $1.8 \pm 1.3$ & \\
\hline \multirow{2}{*}{ IFN-y } & 0 & 39 & $0.4 \pm 0.9$ & ns \\
\hline & 1 & 32 & $0.3 \pm 0.7$ & \\
\hline \multirow[t]{2}{*}{ TNF- $\alpha$} & 0 & 39 & $2.1 \pm 1.9$ & ns \\
\hline & 1 & 32 & $1.9 \pm 1.6$ & \\
\hline \multirow[t]{2}{*}{ VEGF } & 0 & 39 & $40.0 \pm 35.5$ & ns \\
\hline & 1 & 32 & $29.6 \pm 26.0$ & \\
\hline \multirow[t]{2}{*}{ EGF } & 0 & 39 & $471 \pm 69.1$ & ns \\
\hline & 1 & 32 & $46.3 \pm 55.9$ & \\
\hline \multirow[t]{2}{*}{ MCP-1 } & 0 & 39 & $92.2 \pm 89.9$ & ns \\
\hline & 1 & 32 & $72.7 \pm 47.1$ & \\
\hline
\end{tabular}

correlations: IL-2 with IL-4, IL-8, IL-10, IL-1A, IL-1B, and TNF- $\alpha(p=0.000, p=0.016, p=0.000, p=0.000$, $p=0.000$, and $p=0.000$, respectively); IL-6 with IL-1A, TNF- $\alpha$, VEGF, EGF, and MCP-1 ( $p=0.047, p=0.021$, $p=0.000, p=0.001$, and $p=0.027$, respectively); VEGF with EGF and MCP-1 $(p=0.000$ and $p=0.014$, respectively), EGF with $\mathrm{MCP}-1$ ( $p=0.005)$, etc., (Table 4).

After identifying inter-inflammatory biomarker correlations, we wanted to test possible associations with traditional CVD risk factors. Table 5 shows all statistically significant associations identified by univariate analysis. hsCRP shows significant association with female gender ( $p=0,008$ [OR 2,720 for $f$ ) and IL-6 $(p=0,005)$; IL-2 is associated with risk age $(p=0,006)$; IL-4 is reversely associated with total estimated EUROSCORE (\%) and risk age $(p=0,011$ and $p=0.054$, respectively), and significantly associated with $A p o B$ and $\operatorname{Lp}(a)(p=0.006$ and $p=0.036$, respectively). IL-6 is associated with family history for HLP, relative risk, and SBP $(p=0.046)$, as well as with hsCRP, CRP levels $>2 \mathrm{mg} / \mathrm{L}$, and CRP as a category (OR 3.461 for the
$>3 \mathrm{mg} / \mathrm{L}$ category) ( $p=0.005, p=0.016$, and $p=0.001$, respectively). IL-8 is associated with $L p(a)(p=0.044)$ and Acidum uricum $(p=0.008)$ and reversely associated with age $(p=0,005)$ and HTA $(p=0.032)$. IL-10 is also associated with family history for HLP, Chol, and LDL-C ( $p=0.004, p=0.038$, and $p=0.003$ ). IL-1A (same as II-8) shows association with $\operatorname{Lp}(a)(p=0.005)$ and Acidum uricum ( $p=0.038)$, while IL-1B is associated with LDL-C ( $p=0.040)$, and reversely associated with HbA1c $(p=0.050)$. IFN- $\gamma$ shows association with family history for HLP $(p=0.050)$ and $\operatorname{Lp}(a)(p=0.000)$, same as TNF- $\alpha$ ( $p=0.001$ and $p=0.003$, respectively), which is also significantly associated with Chol, LDL-C, ApoB, Lp(a), and Acidum uricum ( $p=0.001, p=0.041$, $p=0.004, p=0.033$, and $p=0.018$, respectively), and reversely associated with age $(p=0.014)$. MCP-1 shows a positive correlation with DM, glucose levels, and $\mathrm{HbA} 1 \mathrm{c}(\mathrm{p}=0.015, \mathrm{p}=0.036$, and $\mathrm{p}=0.003$, respectively). EGF shows a positive correlation with glucose levels $(p=0,013)$.

To test for independently associated variables with each of the studied inflammatory biomarkers, a multivariate analysis was performed using the linear regression model backward conditional (Table 6). hsCRP was reported to be positively correlated with HDL-C and IL-6 ( $p=0.014)$. IL-6 was positively correlated with VEGF and EGF ( $p=0.010$ and $p=0.011$, respectively). Both IL-1A and IL-1B were negatively correlated with $\mathrm{HbA1c}(p=0.029$ and $p=0.026$, respectively). MCP-1 was positively correlated with SBP $(p=0.013)$. VEGF was strongly positively correlated with EGF and vice versa $(p=0,000)$.

To identify independently associated variables with total estimated EUROSCORE risk, surpassing the traditional CVD risk factors that are being used to calculate the risk (age, gender, smoking status, total cholesterol, and SBP), a multivariate linear regression-backward analysis was performed where EUROSCORE risk was used as a single dependent variable, after exclusion of the previously mentioned EUROSCORE identifiers. The analysis revealed 5 traditional risk factors and 7 inflammatory biomarkers

Table 4: Correlations matrix between inflammatory cytokines

\begin{tabular}{|c|c|c|c|c|c|c|c|c|c|c|c|}
\hline & & IL-4 & IL-8 & IL-10 & IL-1A & IL-1B & IFNG & TNFA & VEGF & EGF & MCP-1 \\
\hline \multirow[t]{2}{*}{ IL-2 } & Pearson correlation & $0.482^{\prime \prime}$ & 0.286 & $0.676 "$ & $0.527^{*}$ & $0.443^{\text {th }}$ & 0.225 & $0.592^{* *}$ & & & \\
\hline & Sig. (2-tailed) & 0.000 & 0.016 & 0.000 & 0.000 & 0.000 & 0.059 & 0.000 & & & \\
\hline \multirow[t]{2}{*}{ IL-4 } & Pearson correlation & & $0.299^{*}$ & & $0.409^{\prime \prime}$ & 0.228 & $0.297^{*}$ & $0.317^{*}$ & & & \\
\hline & Sig. (2-tailed) & & 0.011 & & 0.000 & 0.056 & 0.012 & 0.007 & & & \\
\hline \multirow[t]{2}{*}{ IL-6 } & Pearson correlation & & & & $0.237^{*}$ & & & $0.274^{\circ}$ & $0.460^{* *}$ & $0.400^{* \prime}$ & $0.262^{*}$ \\
\hline & Sig. (2-tailed) & & & & 0.047 & & & 0.021 & 0.000 & 0.001 & 0.027 \\
\hline \multirow[t]{2}{*}{ IL-8 } & Pearson correlation & & & & $0.606^{* *}$ & $0.255^{\star}$ & $0.274^{*}$ & $0.355^{* *}$ & & $0.425^{* *}$ & \\
\hline & Sig. (2-tailed) & & & & 0.000 & 0.032 & 0.021 & 0.002 & & 0.000 & \\
\hline \multirow[t]{2}{*}{ IL-10 } & Pearson correlation & & & & $0.461^{*}$ & $0.267^{\star}$ & & $0.747^{*}$ & & & \\
\hline & Sig. (2-tailed) & & & & 0.000 & 0.024 & & 0.000 & & & \\
\hline \multirow[t]{2}{*}{ IL-1A } & Pearson correlation & & & & & 0.452 & 0.384 & $0.553^{* \prime}$ & $0.294^{*}$ & & \\
\hline & Sig. (2-tailed) & & & & & 0.000 & 0.001 & 0.000 & 0.013 & & \\
\hline \multirow{2}{*}{ IL-1B } & Pearson correlation & & & & & & & $0.440^{*}$ & & & \\
\hline & Sig. (2-tailed) & & & & & & & 0.000 & & & \\
\hline \multirow[t]{2}{*}{ IFNG } & Pearson correlation & & & & & & & $0.422^{*}$ & & & \\
\hline & Sig. (2-tailed) & & & & & & & 0.000 & & & \\
\hline \multirow[t]{2}{*}{ VEGF } & Pearson correlation & & & & & & & & & $0.550^{\circ *}$ & $0.291^{*}$ \\
\hline & Sig. (2-tailed) & & & & & & & & & 0.000 & 0.014 \\
\hline \multirow[t]{2}{*}{ EGF } & Pearson correlation & & & & & & & & & & $0.329^{*}$ \\
\hline & Sig. (2-tailed) & & & & & & & & & & 0.005 \\
\hline
\end{tabular}


Table 5: Univariate analysis of inflammatory biomarkers and ASCVD risk identifiers

\begin{tabular}{|c|c|c|}
\hline \multicolumn{3}{|c|}{ Inflammatory biomarker } \\
\hline hsCRP & Beta & Sig \\
\hline Gender (f) & -0.303 & 0.008 (OR 2.720 for f) \\
\hline Risk age & 0.229 & 0.048 \\
\hline HDL-C & 0.236 & 0.042 \\
\hline IL-6 & 0.332 & 0.005 \\
\hline \multicolumn{3}{|l|}{$\frac{\pi L-2}{I L-2}$} \\
\hline HTA (0) & -0.248 & 0.037 \\
\hline Risk age (1) & 0.323 & 0.006 \\
\hline LDL-C & 0.237 & 0.046 \\
\hline IL-4 & 0.482 & 0.000 \\
\hline IL-8 & 0.286 & 0.016 \\
\hline IL-10 & 0.676 & 0.000 \\
\hline IL-1A & 0.527 & 0.000 \\
\hline IL-1B & 0.443 & 0.000 \\
\hline IFN-y & 0.225 & 0.059 \\
\hline TNF- $\alpha$ & 0.592 & 0.000 \\
\hline \multicolumn{3}{|l|}{ IL-4 } \\
\hline Risk (categorical) & -0.301 & 0.011 \\
\hline Risk age & -0.230 & 0.054 \\
\hline ApoB & 0.321 & 0.006 \\
\hline Lp(a) & 0.250 & 0.036 \\
\hline IL-2 & 0.482 & 0.000 \\
\hline IL-8 & 0.299 & 0.011 \\
\hline IL-1A & 0.409 & 0.000 \\
\hline IL-1B & 0.228 & 0.056 \\
\hline IFN-y & 0.297 & 0.012 \\
\hline TNF- $\alpha$ & 0.317 & 0.007 \\
\hline \multicolumn{3}{|l|}{ IL-6 } \\
\hline Gender (f) & -0.304 & 0.010 \\
\hline Family history (1) & 0.238 & 0.046 \\
\hline Relative risk & 0.238 & 0.046 \\
\hline SBP & 0.237 & 0.046 \\
\hline hsCRP & 0.332 & 0.005 \\
\hline $\mathrm{CRP}>2 \mathrm{mg} / \mathrm{L}$ & 0.285 & 0.016 \\
\hline CRP categorical & 0.385 & 0.001 (OR 3.461 for $>3 \mathrm{mg} / \mathrm{L}$ ) \\
\hline IL-A1 & 0.237 & 0.047 \\
\hline TNF- $\alpha$ & 0.274 & 0.021 \\
\hline MCP-1 & 0.262 & 0.027 \\
\hline VEGF & 0.460 & 0.000 \\
\hline EGF & $0 . .400$ & 0.001 \\
\hline \multicolumn{3}{|l|}{ IL-8 } \\
\hline Age & -0.333 & 0.005 \\
\hline HTA & -0.255 & 0.032 \\
\hline $\operatorname{Lp}(\mathrm{a})$ & 0.240 & 0.044 \\
\hline Acidum uricum & 0.311 & 0.008 \\
\hline IL-2 & 0.286 & 0.016 \\
\hline IL-4 & 0.299 & 0.011 \\
\hline IL-1A & 0.606 & 0.000 \\
\hline IL-1B & 0.255 & 0.032 \\
\hline IFN-Y & 0.274 & 0.021 \\
\hline TNF- $\alpha$ & 0.355 & 0.002 \\
\hline VEGF & 0.225 & 0.059 \\
\hline EGF & 0.425 & 0.000 \\
\hline $\mathrm{IL}-10$ & & \\
\hline Family history & 0.341 & 0.004 \\
\hline HTA & -0.225 & 0.059 \\
\hline Chol & 0.247 & 0.038 \\
\hline LDL-C & 0.350 & 0.003 \\
\hline IL-2 & 0.676 & 0.000 \\
\hline IL-1A & 0.461 & 0.000 \\
\hline IL-1B & 0.267 & 0.024 \\
\hline TNF- $\alpha$ & 0.747 & 0.000 \\
\hline $\mathrm{IL}-1 \mathrm{~A}$ & & \\
\hline$L p(a)$ & 0.329 & 0.005 \\
\hline $\mathrm{HbA} 1 \mathrm{c}$ & -0.229 & 0.055 \\
\hline Acidum uricum & 0.247 & 0.038 \\
\hline IL-2 & 0.527 & 0.000 \\
\hline IL-4 & 0.409 & 0.000 \\
\hline IL-6 & 0.237 & 0.047 \\
\hline IL-8 & 0.606 & 0.000 \\
\hline IL-10 & 0.461 & 0.000 \\
\hline IL-1B & 0.452 & 0.000 \\
\hline INF-y & 0.384 & 0.001 \\
\hline TNF- $\alpha$ & 0.553 & 0.000 \\
\hline VEGF & 0.294 & 0.013 \\
\hline IL-1B & & \\
\hline LDL-C & 0.244 & 0.040 \\
\hline $\mathrm{HbA} 1 \mathrm{c}$ & -0.233 & 0.050 \\
\hline IL-2 & 0.443 & 0.000 \\
\hline IL-4 & 0.228 & 0.056 \\
\hline IL-8 & 0.255 & 0.032 \\
\hline IL-10 & 0.267 & 0.024 \\
\hline IL-1A & 0.452 & 0.000 \\
\hline TNF- $\alpha$ & 0.440 & 0.000 \\
\hline
\end{tabular}

Table 5: (Continued)

\begin{tabular}{|c|c|c|}
\hline \multicolumn{3}{|c|}{ Inflammatory biomarker } \\
\hline \multicolumn{3}{|c|}{ IFN-Y } \\
\hline Family history & 0.234 & 0.050 \\
\hline $\operatorname{Lp}(a)$ & 0.672 & 0.000 \\
\hline IL-2 & 0.225 & 0.059 \\
\hline IL-4 & 0.297 & 0.012 \\
\hline IL-8 & 0.274 & 0.021 \\
\hline IL-1A & 0.384 & 0.001 \\
\hline TNF- $\alpha$ & 0.422 & 0.000 \\
\hline \multicolumn{3}{|l|}{ TNF- $\alpha$} \\
\hline Age & -0.290 & 0.014 \\
\hline Family history & 0.375 & 0.001 \\
\hline HTA & -0.230 & 0.054 \\
\hline Chol & 0.243 & 0.041 \\
\hline LDL-C & 0.336 & 0.004 \\
\hline ApoB & 0.253 & 0.033 \\
\hline $\operatorname{Lp}(a)$ & 0.351 & 0.003 \\
\hline Acidum uricum & 0.280 & 0.018 \\
\hline IL-2 & 0.592 & 0.000 \\
\hline IL-4 & 0.317 & 0.007 \\
\hline IL-6 & 0.274 & 0.021 \\
\hline IL-8 & 0.355 & 0.002 \\
\hline IL-10 & 0.747 & 0.000 \\
\hline IL-1A & 0.553 & 0.000 \\
\hline IL-1B & 0.440 & 0.000 \\
\hline \multicolumn{3}{|l|}{ MCP-1 } \\
\hline DM & 0.288 & 0.015 \\
\hline SBP & 0.231 & 0.052 \\
\hline $\mathrm{Gl}$ & 0.249 & 0.036 \\
\hline $\mathrm{HbA1c}$ & 0.352 & 0.003 \\
\hline IL-6 & 0.262 & 0.027 \\
\hline VEGF & 0.291 & 0.014 \\
\hline EGF & 0.329 & 0.005 \\
\hline \multicolumn{3}{|l|}{ VEGF } \\
\hline IL-6 & 0.460 & 0.000 \\
\hline IL-8 & 0.225 & 0.059 \\
\hline IL-1A & 0.294 & 0.013 \\
\hline MCP-1 & 0.291 & 0.014 \\
\hline EGFO & 0.550 & 0.000 \\
\hline \multicolumn{3}{|l|}{ EGF } \\
\hline $\mathrm{Gl}$ & 0.292 & 0.013 \\
\hline IL-6 & 0.400 & 0.001 \\
\hline IL-8 & 0.425 & 0.000 \\
\hline MCP-1 & 0.329 & 0.005 \\
\hline VEGF & 0.550 & 0.000 \\
\hline
\end{tabular}

to be independently, significantly associated with total CVD risk (Table 7).

\section{ability}

\section{Inflammatory biomarkers - discrimination}

The inflammatory biomarker discrimination ability was tested against the patients' SCORE risk profile using "receiver operating characteristic" (ROC) curves. The discriminatory function of the inflammatory biomarkers corresponds with the SCORE-risk profile severity; therefore, they do not have any statistically significant discrimination ability in patients at low-tomoderate risk. IL-4, with its low values, was the only inflammatory biomarker to show a statistically significant discriminatory function in patients with high-to-very high risk (0.323; $p=0.021$ ) (Figure 1).

The inflammatory biomarker discrimination ability was tested against the patients' hsCRP levels as well. IL-6, VEGF, and EGF were reported to have statistically significant discriminatory functions in patients with hsCRP levels $<1 \mathrm{mg} / \mathrm{dl}$, with low values as determinants $(0.284 ; p=0.004 ; 0.359 ; p=0.059$; and 0.350; $p=0.044$, respectively) (Figure 2).

IL-6 was shown to have a statistically significant discriminatory function in patients with hsCRP levels 
Table 6: Multivariate analysis of independently associated variables (Linear regression model)

\begin{tabular}{|c|c|c|c|c|}
\hline Dependent variable & Variables in the model & Beta & $\mathrm{t}$ & Sig \\
\hline hsCRP & HDL-C & 0.272 & 2.518 & 0.014 \\
\hline $\mathrm{R}$ square $.222 ; p=0.001$ & $\mathrm{IL}-6$ & 0.285 & 2.524 & 0.014 \\
\hline $\mathrm{IL}-2$ & RISK age & 0.174 & 2.321 & 0.023 \\
\hline \multirow[t]{3}{*}{ R square $.650 ; p=0.000$} & $\mathrm{IL}-4$ & 0.352 & 4.527 & 0.000 \\
\hline & $\mathrm{IL}-10$ & 0.537 & 6.997 & 0.000 \\
\hline & IL-1B & 0.233 & 3.035 & 0.003 \\
\hline IL-4 & Risk categorical & -0.365 & -4.314 & 0.000 \\
\hline \multirow[t]{6}{*}{$R$ square $0.650 ; p=0.000$} & TG & 0.273 & 3.085 & 0.003 \\
\hline & ApoA1 & 0.149 & 1.748 & 0.085 \\
\hline & ApoB & 0.299 & 3.323 & 0.001 \\
\hline & $\mathrm{IL}-2$ & 0.530 & 4.262 & 0.000 \\
\hline & $\mathrm{IL}-10$ & -0.391 & -3.353 & 0.0 .001 \\
\hline & IL-1A & 0.388 & 3.714 & 0.000 \\
\hline IL-6 & female $0 /$ male 1 & $-0.164)$ & -1.781 & 0.080 \\
\hline \multirow[t]{5}{*}{$R$ square $0.585 ; p=0.000$} & SBP & 0.345 & 3.932 & 0.000 \\
\hline & hsCRP & 0.291 & 3.218 & 0.002 \\
\hline & TNF- $\alpha$ & 0.216 & 2.455 & 0.017 \\
\hline & VEGF & 0.282 & 2.664 & 0.010 \\
\hline & EGF & 0.276 & 2.628 & 0.011 \\
\hline IL-8 & Age & -0.222 & -2.546 & 0.013 \\
\hline \multirow[t]{3}{*}{ R square $292.180 ; p=0.000$} & Acidum uricum & 0.185 & 2.102 & 0.039 \\
\hline & IL-1A & 0.468 & 5.208 & 0.000 \\
\hline & EGF & 0.268 & 3.056 & 0.003 \\
\hline IL-10 & $\mathrm{IL}-2$ & 0.482 & 5.039 & 0.000 \\
\hline \multirow[t]{3}{*}{$R$ square $2.591 ; p=0.000$} & IL-4 & -0.163 & -2.061 & 0.043 \\
\hline & IL-1B & -0.168 & -2.108 & 0.039 \\
\hline & TNF- $\alpha$ & 0.587 & 6.619 & 0.000 \\
\hline IL-1A & $\operatorname{Lp}(a)$ & 0.169 & 2.085 & 0.041 \\
\hline \multirow{5}{*}{$R$ square $0.169 ; p=0.000$} & $\mathrm{HbA1c}$ & -0.176 & -2.237 & 0.029 \\
\hline & IL-2 & 0.286 & 2.615 & 0.011 \\
\hline & IL-8 & 0.404 & 4.790 & 0.000 \\
\hline & $\mathrm{IL}-10$ & 0.190 & 1.799 & 0.077 \\
\hline & VEGF & 0.149 & 1.852 & 0.069 \\
\hline IL-1B & HbA1c & -0.233 & -2.281 & 0.026 \\
\hline \multirow[t]{3}{*}{$R$ square $6.662 ; p=0.000$} & IL-2 & 0.463 & 3.313 & 0.001 \\
\hline & $\mathrm{IL}-10$ & -0.364 & -2.182 & 0.033 \\
\hline & TNFA & 0.410 & 2.655 & 0.010 \\
\hline IFN-y & $\operatorname{Lp}(\mathrm{a})$ & 0.646 & 7.463 & 0.000 \\
\hline$R$ square $8.491 ; p=0.000$ & IL-2 & 0.179 & 2.082 & 0.041 \\
\hline TNF- $\alpha$ & Age & -0.115 & -1.743 & 0.086 \\
\hline \multirow[t]{5}{*}{$R$ square $28.315 ; p=0.000$} & $\operatorname{Lp}(\mathrm{a})$ & 0.287 & 4.465 & 0.000 \\
\hline & Acidum uricum & 0.114 & 1.799 & 0.077 \\
\hline & IL-6 & 0.122 & 1.904 & 0.061 \\
\hline & $\mathrm{IL}-10$ & 0.648 & 9.882 & 0.000 \\
\hline & IL-1B & 0.181 & 2.790 & 0.007 \\
\hline MCP-1 & SBP & 0.267 & 2.551 & 0.013 \\
\hline \multirow[t]{3}{*}{$R$ square $30469.901 ; p=0.000$} & $\mathrm{HbA1c}$ & 0.325 & 3.119 & 0.003 \\
\hline & VEGF & 0.229 & 1.857 & 0.068 \\
\hline & EGF & 0.230 & 1.841 & 0.070 \\
\hline VEGF & IL-6 & 0.286 & 2.725 & 0.008 \\
\hline$R$ square $13133.260 ; p=0.000$ & EGF & 0.436 & 4.154 & 0.000 \\
\hline EGF & IL-6 & 0.169 & 1.741 & 0.086 \\
\hline \multirow[t]{4}{*}{$R$ square $29504.865 ; p=0.000$} & IL-8 & 0.371 & 3.429 & 0.001 \\
\hline & IL-1A & $-0.223)$ & $-2.038)$ & 0.046 \\
\hline & $\mathrm{Gl}$ & 0.304 & 3.500 & 0.001 \\
\hline & VEGF & 0.477 & 4.841 & 0.000 \\
\hline
\end{tabular}

$>3 \mathrm{mg} / \mathrm{dl}$, with high values as determinants $(0.776$; $\mathrm{p}=0.000$ ) (Figure 3).

Table 7: Variables independently associated with EUROSCORE risk. after exclusion of identifiers of EUROSCORE (age. gender. smoking. total cholesterol. and SBP). with multivariate linear regression-backward. mean square of the model 66.565 ; sig 0.000

\begin{tabular}{lllll}
\hline EUROSCORE (\%) & Variables in the model & Beta & $\mathrm{t}$ & $\mathrm{Sig}$ \\
\hline & (Constant) & & -4.637 & 0.000 \\
& LDL-C (mmol/L) & 0.439 & 3.314 & 0.002 \\
HDL-C (mmol/L) & -0.176 & -1.995 & 0.051 \\
& TG $(\mathrm{mmol} / \mathrm{L})$ & 0.335 & 3.585 & 0.001 \\
& ApoB $(\mathrm{mg} / \mathrm{dl})$ & 0.720 & 5.451 & 0.000 \\
& HbA1c $(\%)$ & 0.466 & 5.496 & 0.000 \\
hsCRP $(\mathrm{mg} / \mathrm{dl})$ & 0.190 & 2.085 & 0.042 \\
IL-4 & -0.498 & -4.903 & 0.000 \\
& IL-6 & 0.475 & 4.520 & 0.000 \\
IL-10 & -0.242 & -2.432 & 0.018 \\
VEGF & -0.240 & -2.197 & 0.032 \\
& IL-1A & 0.491 & 4.278 & 0.000 \\
EGF & -0.232 & -2.290 & 0.026 \\
\hline
\end{tabular}

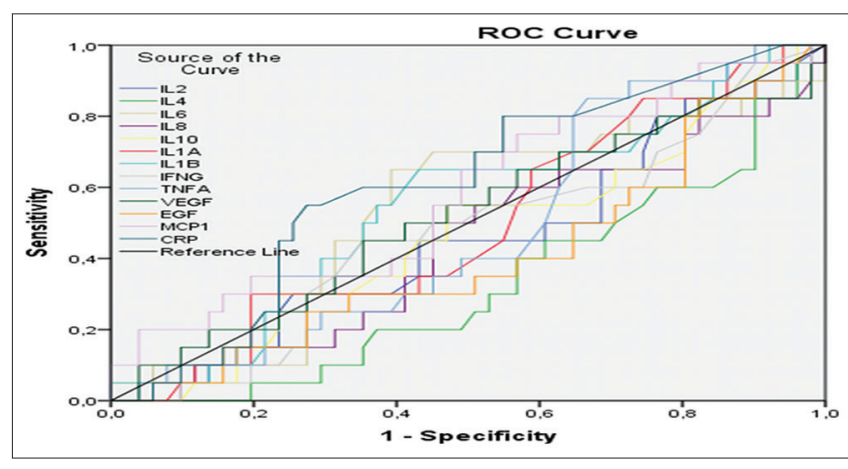

\begin{tabular}{|c|c|c|c|c|c|}
\hline \multirow[t]{2}{*}{ Test result variable(s) } & \multirow[t]{2}{*}{ Area } & \multirow[t]{2}{*}{ Std. error ${ }^{\mathrm{a}}$} & \multirow[t]{2}{*}{ Asymptotic Sig. ${ }^{b}$} & \multicolumn{2}{|c|}{ Asymptotic $95 \% \mathrm{Cl}$} \\
\hline & & & & $\begin{array}{l}\text { Lower } \\
\text { bound }\end{array}$ & $\begin{array}{l}\text { Upper } \\
\text { bound }\end{array}$ \\
\hline hsCRP & 0.610 & 0.072 & 0.150 & 0.470 & 0.751 \\
\hline IL-2 & 0.453 & 0.076 & 0.544 & 0.304 & 0.603 \\
\hline IL-4 & 0.323 & 0.068 & 0.021 & 0.189 & 0.456 \\
\hline IL-6 & 0.538 & 0.078 & 0.623 & 0.384 & 0.691 \\
\hline IL-8 & 0.438 & 0.076 & 0.421 & 0.289 & 0.587 \\
\hline IL-10 & 0.468 & 0.076 & 0.673 & 0.319 & 0.616 \\
\hline VEGF & 0.516 & 0.079 & 0.838 & 0.361 & 0.670 \\
\hline IFN-y & 0.470 & 0.076 & 0.692 & 0.321 & 0.618 \\
\hline TNF- $\alpha$ & 0.481 & 0.072 & 0.803 & 0.340 & 0.621 \\
\hline IL-1A & 0.493 & 0.074 & 0.924 & 0.348 & 0.637 \\
\hline $\mathrm{IL}-1 \mathrm{~B}$ & 0.549 & 0.076 & 0.523 & 0.401 & 0.697 \\
\hline MCP-1 & 0.580 & 0.076 & 0.295 & 0.431 & 0.730 \\
\hline EGF & 0.404 & 0.075 & 0.213 & 0.257 & 0.552 \\
\hline
\end{tabular}

Figure 1: ROC-curves for inflammatory biomarkers in patients with high and very high EUROSCORE risk

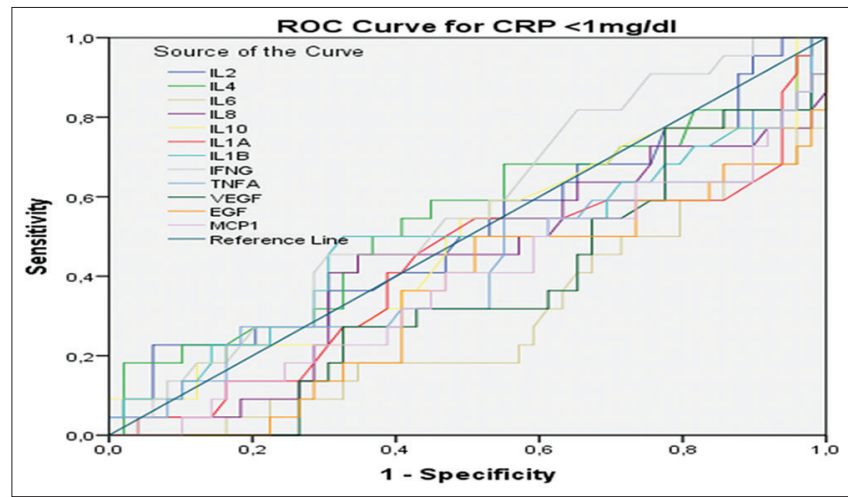

\begin{tabular}{lcllll}
\hline Test result variables & Area & Std. error $^{\mathrm{a}}$ & Sig. $^{\mathrm{b}}$ & \multicolumn{2}{c}{ Asymptotic $95 \% \mathrm{Cl}$} \\
\cline { 5 - 6 } & & & & Lower bound & Upper bound \\
\hline IL-2 & 0.513 & 0.078 & 0.867 & 0.360 & 0.665 \\
IL-4 & 0.538 & 0.079 & 0.606 & 0.383 & 0.694 \\
IL-6 & 0.284 & 0.066 & 0.004 & 0.155 & 0.413 \\
IL-8 & 0.434 & 0.077 & 0.377 & 0.283 & 0.585 \\
IL-10 & 0.482 & 0.077 & 0.808 & 0.330 & 0.633 \\
IL-1A & 0.416 & 0.078 & 0.260 & 0.264 & 0.568 \\
IL-1B & 0.481 & 0.082 & 0.799 & 0.321 & 0.641 \\
IFN-Y & 0.570 & 0.071 & 0.351 & 0.430 & 0.709 \\
TNF- $\alpha$ & 0.422 & 0.079 & 0.293 & 0.267 & 0.577 \\
VEGF & 0.359 & 0.069 & 0.059 & 0.224 & 0.494 \\
EGF & 0.350 & 0.072 & 0.044 & 0.210 & 0.490 \\
MCP-1 & 0.386 & 0.075 & 0.126 & 0.240 & 0.532 \\
\hline
\end{tabular}

Figure 2: ROC-curves for inflammatory biomarkers in patients with $h s C R P<1 \mathrm{mg} / \mathrm{dL}$

\section{hsCRP and IL-6}

Tables 8 and 9 show statistically significant correlations of hsCRP and IL-6. hsCRP is positively

Table 8: hsCRP significant correlations

\begin{tabular}{llllll}
\hline Control variable & female 0/ male 1 & RISK age & HDL-C & IL-6 \\
\hline hsCRP & Correlation & -0.303 & 0.229 & 0.236 & 0.332 \\
& Significance (2-tailed) & 0.008 & 0.048 & 0.042 & 0.005 \\
df & 73 & 73 & 73 & 73 \\
\hline
\end{tabular}




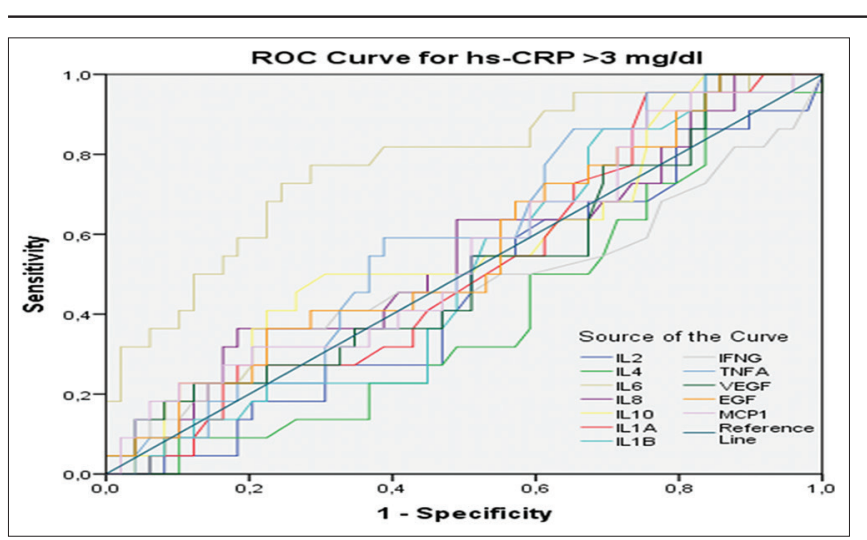

\begin{tabular}{llllll}
\hline Test result variables & Area & Std. error $^{\mathrm{a}}$ & Asymptotic Sig. $^{b}$ & \multicolumn{2}{c}{ Asymptotic 95\% Cl } \\
\cline { 5 - 6 } & & & & Lower bound & Upper bound \\
\hline IL-2 & 0.451 & 0.072 & 0.514 & 0.310 & 0.593 \\
IL-4 & 0.398 & 0.070 & 0.173 & 0.262 & 0.535 \\
IL-6 & 0.776 & 0.061 & 0.000 & 0.656 & 0.897 \\
IL-8 & 0.542 & 0.075 & 0.572 & 0.396 & 0.689 \\
IL-10 & 0.564 & 0.075 & 0.391 & 0.418 & 0.710 \\
IL-1A & 0.514 & 0.071 & 0.847 & 0.375 & 0.654 \\
IL-1B & 0.505 & 0.070 & 0.945 & 0.367 & 0.643 \\
IFN-y & 0.477 & 0.081 & 0.761 & 0.318 & 0.637 \\
TNF- $\alpha$ & 0.579 & 0.069 & 0.291 & 0.443 & 0.714 \\
VEGF & 0.511 & 0.075 & 0.881 & 0.364 & 0.658 \\
EGF & 0.557 & 0.073 & 0.448 & 0.413 & 0.700 \\
MCP-1 & 0.547 & 0.074 & 0.526 & 0.402 & 0.693 \\
\hline
\end{tabular}

Figure 3. ROC-curves for inflammatory biomarkers in patients with $h s C R P>3 \mathrm{mg} / \mathrm{dL}$

correlated with female gender, risk age, HDL-C, and IL-6 ( $p=0.008, p=0.048, p=0.042$, and $p=0.005)$ (Table 8). IL-6 is positively correlated with relative risk, risk age, and hsCRP $(p=0.019, p=0.023$, and $\mathrm{p}=0,000)$ (Table 9).

Table 9: IL-6 significant correlations (nonparametric correlations)

\begin{tabular}{lllll}
\hline Control variable & Relative risk & Risk age & hsCRP \\
\hline IL-6 Correlation & 0.278 & 0.270 & $0.443^{*}$ \\
& Significance (2-tailed) & 0.019 & 0.023 & 0.000 \\
df & 73 & 73 & 73 \\
\hline "With nonparametric tests also. & & &
\end{tabular}

To determine any "cut-off" value of IL-6, we performed a stepwise discriminatory testing and we found that IL-6 levels $>1 \mathrm{pg} / \mathrm{ml}$ demonstrate a significant discriminatory function. Table 10 shows all statistically significant correlations of IL-6 levels > $1 \mathrm{pg} / \mathrm{ml}$ and CVD risk factors identified by univariate binary logistic regression analysis. IL-6 levels above $1 \mathrm{pg} / \mathrm{ml}$ are correlated with female gender, age $>55$ years, EUROSCORE $(\%) \geq 3$, risk age, SBP, hsCRP $>2 \mathrm{mg} / \mathrm{L}$, and IL-2 $(p=0.025, p=0.013, p=0.025$, $p=0.011, p=0.026, p=0.046$, and $p=0.018$, respectively).

Table 10: Univariate binary logistic regression analysis of IL-6 $>1 \mathrm{pg} / \mathrm{ml}$ and CVD risk factors

\begin{tabular}{llll}
\hline IL-6 $>1 \mathrm{pg} / \mathrm{ml}$ & & & \\
\hline Variable & Wald & OR $(95 \% \mathrm{Cl})$ & $\mathrm{Sig}$ \\
\hline Gender (f) & 5.051 & $3.055(1.154-8.088)$ & 0.025 \\
Age $>55 \mathrm{y}$ & 6.131 & $3.562(1.303-9.739)$ & 0.013 \\
DM & 3.483 & $3.808(.935-15.504)$ & 0.062 \\
EUROSCORE $\geq 3$ & 5.012 & $3.087(1.151-8.284)$ & 0.025 \\
Risk age & 6.459 & & 0.011 \\
SBP $(\mathrm{mmHg})$ & 6.965 & $3.030(1.143-8.036)$ & 0.026 \\
hsCRP $>2 \mathrm{mg} / \mathrm{L}$ & 3.978 & $2.654(1.017-6.925) 4$ & 0.046 \\
IL-2 & 5.595 & & 0.018 \\
\hline
\end{tabular}

To identify independently associated variables with IL-6 levels above $1 \mathrm{pg} / \mathrm{ml}$, a multivariate linear regression-backward analysis was performed using IL-6 $>1 \mathrm{pg} / \mathrm{ml}$ as a single dependent variable. The analysis revealed female gender, age above 55 years, HTA (or SBP > $140 \mathrm{mmHg}$ ), hsCRP levels above $2 \mathrm{mg} / \mathrm{L}$, and IL-2 to be independently, significantly associated with IL-6 > 1 pg/ml (Table 11).

Table 11: Variables independently associated with IL-6 > $1 \mathrm{pg} / \mathrm{ml}$. with multivariate logistic regression model (backward conditional). Chi-square of the model 32.169; $p=0.000$; percent of correct prediction $78.9 \%$

\begin{tabular}{lllllll}
\hline B & & Wald & Sig. & $\operatorname{Exp}(B)$ & \multicolumn{2}{c}{ 95\% C.I. for EXP(B) } \\
\cline { 6 - 7 } & & & & & Lower & Upper \\
\hline Gender (f) & -1.528 & 4.960 & 0.026 & 0.217 & 0.057 & 0.833 \\
Age $>55$ & 1.595 & 6.091 & 0.014 & 4.928 & 1.389 & 17.492 \\
SBP $>140 \mathrm{mmHg}$ & 1.757 & 6.433 & 0.011 & 5.797 & 1.491 & 22.539 \\
hsCRP $>2 \mathrm{mg} / \mathrm{L}$ & 1.064 & 2.826 & 0.093 & 2.897 & 0.838 & 10.014 \\
IL-2 & 1.252 & 9.757 & 0.002 & 3.497 & 1.594 & 7.672 \\
Constant & -3.571 & 10.673 & .001 & 0.028 & & \\
\hline
\end{tabular}

\section{Discussion}

The aim of this study was to test for a possible correlation between the cardiovascular risk profile of healthy subjects at medium-to-high CVD risk and various inflammatory markers, and assess whether IL-6 can serve as a better inflammatory marker than CRP in CVD risk prediction. To achieve our goals, we included 75 adult ambulatory patients without an established CVD, obtaining the levels of various biochemical and inflammatory markers and establishing the subjects' complete risk profile. Patients were analyzed by three indicators: HLP, DM, and a hsCRP value $>2 \mathrm{mg} / \mathrm{L}$. The previous was based on: 1 . The well-established role of HLP as a CVD risk factor [18]; 2. The reciprocal relationship between DM and CVD; a hypothesis that the two diseases share common antecedents, supported by the recently discovered shared molecular drivers, pathways and gene subnetworks [19], [20], [21]; and 3. hsCRP values above $2 \mathrm{mg} / \mathrm{L}$ are considered as an indicator of increased CVD risk [10], [11], [22].

Our study population showed a total estimated EUROSCORE (\%) of 3,5, with a relative risk of 2,7, meaning a 3-fold increased CVD risk compared to an equivalent population with normal risk factor values. More than half of the study population $(62,7 \%)$ had a risk age above their actual biological age. Testing for significant associations between the patients' subgroups revealed two interesting correlations; DM was positively correlated with MCP-1 levels $(130,2 \pm 102,8$ vs. $73,9 \pm 63,6, p=0.050)$ and hsCRP levels $>2 \mathrm{mg} / \mathrm{L}$ were positively correlated with IL-6 levels $(1.9 \pm 2.2$ vs. $1.0 \pm 0.6, p=0.017)$. Diabetic 2518GG-carriers (a polymorphism in the gene that regulates MCP-1 expression) have been found to have elevated circulating MCP-1 levels and increased insulin 
resistance [23]. The connection between hsCRP and IL-6 will be addressed later in the article.

Compared to males, females showed higher levels of hsCRP and IL-6 (4.2 \pm 4.7 vs. $1.9 \pm$ 1.5 and $1.9 \pm 2.1$ vs. $0.9 \pm 0.5$, with both parametric and nonparametric tests $(p=0.008 / p=0.002$ and $p=0.010 / p=0.003$, respectively). Gender and race have been shown to affect hsCRP levels, with higher hsCRP concentrations found in females [24]. Milan-Mattos et al. (2019) evaluated IL-6 and hsCRP levels in healthy men and women of different age groups. Women presented with stronger correlations, compared to men, for both IL-6 and hsCRP, and the 51-60 age group was the key point for the increase [25].

We used several statistical methods to identify correlations between the inflammatory markers and traditional risk factors, as well as amongst the inflammatory markers themselves. Numerous statistically significant correlations were detected (Table 4), adding to the principle of the "inflammatory cascade." TNF- $\alpha$ is one of the most potent proinflammatory cytokines appearing early in the inflammatory response, which despite its own actions on different signal transduction pathways, enhances the inflammatory response by stimulating IL-1A, IL-1B, IL-8, and MCP-1 synthesis in macrophages and endothelial cells [26]. IL-6 is another major cytokine that acts early in the inflammatory response, stimulating hepatic production of acute-phase proteins, including CRP [27]. IL-6 also acts by inducing VEGF and EGF synthesis in endothelial cells, both major regulators of angiogenesis and vasculogenesis [28]. EGF is thought to exert its angiogenic effects on vascular endothelial cells by stimulating the autocrine secretion of VEGF [29]. Plenty of evidence can be found in the scientific literature regarding associations between different inflammatory cytokines that can be used to explain the correlations obtained in our study.

Statistically significant associations of every studied inflammatory biomarker, obtained with both univariate and multivariate analysis, are shown in Tables 5 and 6 . We will only present several correlations with subsequent scientific evidence. hsCRP was reported to be positively correlated with HDL-C and IL-6 ( $p=0,014)$. Given that lower HDL-C levels are considered a CVD risk factor, its proportional correlation with hsCRP, which is a pro-inflammatory marker, is unexpected. However, there are recent findings stating that HDL may become dysfunctional in some disease states, such as atherosclerosis. Activation of an acute phase response (characterized with increased hsCRP levels) leads to HDL changes, such as loss of apoA-I and paraoxonase (PON) and incorporation of acute-phase proteins, which in turn reduces the HDL anti-oxidant capacity. Accordingly, higher HDL-C concentrations are needed to counterbalance the increased hsCRP levels [30], [31], [32]. Further studies are needed. IL-4 was negatively correlated with total estimated EUROSCORE (\%) and risk age ( $p=0.011$ and $p=0.054$, respectively), and positively correlated with $A p o B$ and $\operatorname{Lp}(a)(p=0.006$ and $p=0.036$, respectively). IL-4 is a cytokine with varying pro/anti-inflammatory actions depending on the overall state of the subject [33]. In healthy subjects, IL-4 exerts anti-inflammatory actions by suppressing monocyte and macrophage pro-inflammatory cytokine production, explaining its negative correlation with total CVD risk [34]. However, recent in vitro and in vivo studies have provided evidence that IL-4 exerts proinflammatory effects on the vascular endothelium in patients with disturbed lipid balance, such as increased levels of $A p o B$ and $L p(a)$ [35]. IL-8, IL-1A, IFN- $\gamma$, and TNF- $\alpha$ all showed positive correlations with $\operatorname{Lp}(a)(p=0.044, p=0.005, p=0.000$, and $p=0.003$, respectively). $L p(a)$ promotes differentiation of the pro-inflammatory, M1-type macrophages that secrete numerous pro-inflammatory cytokines, such as IL-1A and IL-1B, IL-6, IL-8, TNF- $\alpha$, and IFN- $\gamma$, which is considered to be one of the mechanisms responsible for its proatherosclerotic potential [36]. IL-8, IL-1A, and TNF- $\alpha$ were all positively correlated with Acidum uricum ( $p=0.008, p=0.038$, and $p=0.018$, respectively). Recent studies have shown that high levels of Acidum uricum predict HTA and CV event development, possibly through inflammation. Tissue damage releases endogenous substances, including Acidum uricum, which signals danger and stimulates inflammation. The secreted Acidum uricum then enters the vascular smooth muscle cells stimulating CRP and MCP-1 release. It also penetrates human mononuclear cells, where it stimulates IL-1, IL-8, and TNF- $\alpha$ production [37]. MCP-1 showed a positive correlation with DM, glucose levels, and $\operatorname{HbA} 1 c(p=0.015, p=0.036$, and $p=0.003$, respectively). Circulating MCP-1 levels have been found to be significantly increased in Type 2 diabetic patients, carriers of the MCP-1 G-2518 gene variant. High glucose levels stimulate endothelial cells to increase MCP-1 release, as well as increase basal expression of vascular cell adhesion molecule-1 (VCAM-1), leading to a synergistic enhancement of the monocyte-endothelial cell interaction [38].

A multivariate linear regression-backward analysis combining the traditional risk factors and inflammatory biomarkers (surpassing the CVD indicators used to calculate EUROSCORE risk) revealed 12 markers to be significantly, independently associated with CVD risk - LDL-C, TG, ApoB, HbA1c, hsCRP, IL-6, and IL-1A as markers of higher, and HDLC, IL-4, II-10, VEGF, and EGF as markers of lower CVD risk (Table 7).

We tested the inflammatory biomarker discrimination abilities using ROC curves, according to the previously discussed subjects' subgroups. The discriminatory function of the inflammatory biomarkers corresponds with the SCORE-risk profile severity and hsCRP levels; they did not show any statistically 
significant discrimination ability regarding the presence/ absence of DM. IL-4, with its low values, was the only inflammatory biomarker to show a statistically significant discriminatory function in patients at high-to-very high CVD risk (0.323; $p=0.021)$. Its anti-inflammatory potential was previously discussed. IL-6 was shown to have a statistically significant discriminatory function in patients with hsCRP levels $>3 \mathrm{mg} / \mathrm{dl}$, with high values as determinants $(0.776 ; p=0.000)$.

Every single correlation test, univariate, or multivariate analysis, we did show a statistically significant positive association between hsCRP and IL-6. Even ROC curves testing discriminatory function of different inflammatory markers identified IL-6 as a major, proportional determinant of hsCRP levels. The previous can be explained by the inflammatory pathway of hsCRP - hsCRP is the final downstream biomarker of a complex cascade which includes intermediate IL-6 and upstream IL-1 signaling pathways. IL-6 binds directly to the cellular membrane-bound IL-6 receptor, expressed on hepatocytes and several white blood cells, forming a signaling complex that leads to acutephase protein production, including hsCRP synthesis. On the other hand, part of the circulating IL- 6 binds to the soluble portion of the IL-6 receptors, forming a binary complex, which has been shown to have independent pro-inflammatory activities, additional to the hsCRP synthesis induction [39].

Like hsCRP, IL-6 levels, measured in apparently healthy populations, also predict future vascular risk, an observation made in more than 25 prospective epidemiologic cohorts worldwide. For each SD increase in log IL-6, there is a $25 \%$ increase in the risk of future vascular events $(\mathrm{RR} 1.25,95 \% \mathrm{Cl} 1.19$ 1.32). IL-6 levels have been shown to correlate with endothelial dysfunction, arterial stiffness, and extent of sub-clinical atherosclerosis [17].

Taking into consideration the previous, we wanted to test whether IL-6 can be used as a sole inflammatory marker instead of hsCRP. We chose a cut-off IL-6 value of $1 \mathrm{pg} / \mathrm{ml}$ - given the results obtained with our study and the source scientific data. A univariate analysis showed that IL-6 levels above $1 \mathrm{pg} / \mathrm{ml}$ are significantly, positively correlated with female gender, age $>55$ years, EUROSCORE $(\%) \geq 3$, risk age, SBP, hsCRP $>2 \mathrm{mg} / \mathrm{L}$, and IL-2 ( $p=0.025$, $p=0.013, p=0.025, p=0.011, p=0.026, p=0.046$, and $p=0.018$, respectively). Except for total CVD risk and risk age, the same variables were identified as independently associated variables with IL-6 levels above $1 \mathrm{pg} / \mathrm{ml}$, with a multivariate linear regressionbackward analysis. Our results were in accordance with current scientific reports [40], [41], [42], [43], [44].

\section{Limitations of the study}

Due to the small sample size, the number of subjects in each subgroup is not equal. We think that this is the main reason for omitting possible statistically significant associations that would appear with larger sample size and proportional subject distribution.

\section{Conclusion}

In this cohort of healthy subjects, total CVD risk was strongly and independently associated with several circulating markers of inflammation. hsCRP appears to be in a strong reciprocal relationship with IL-6. IL-6 was significantly and independently correlated with several traditional risk factors, as well as with hsCRP and IL-2. Therefore, IL-6 may have a potential role as a mediator between cardiovascular risk factors and several biological mechanisms for CVD not only in diagnostic but also in therapeutic goals as well.

\section{Ethical approval}

The Clinical Studies and Ethics Committee of UKIM-Faculty of Medicine, Skopje, Republic of Macedonia approved this study; Reference number: 03-3152/9

\section{Contributorship}

MV and KM designed, conducted, and supervised the research; analyzed data and performed the statistical analysis; and wrote the manuscript. AV designed and conducted the research; analyzed data and performed the statistical analysis; and wrote the manuscript. $A D$ and GP supervised the research; $A E$, $S D$, and $A D$ conducted the research. All authors had primary responsibility for the final content.

\section{References}

1. Moran AE, Forouzanfar MH, Roth GA, Mensah GA, Ezzati M Murray CJ, et al. Temporal trends in ischemic heart disease mortality in 21 world regions, 1980 to 2010: The global burden of disease 2010 study. Circulation. 2014;129(14):1483-92. https:// doi.org/10.1161/circulationaha.113.004042

PMid:24573352

2. Piepoli MF, Hoes AW, Agewall S, Albus C, Brotons C, Catapano AL, et al. 2016 European guidelines on cardiovascular disease prevention in clinical practice: The sixth joint task force of the European society of cardiology and other societies on cardiovascular disease prevention in clinical practice (constituted by representatives of 10 societies and by invited experts) developed with the special contribution of the European association for cardiovascular prevention and rehabilitation (EACPR). Eur Heart J. 2016;37(29):2315-81. https://doi. org/10.1007/s12529-016-9583-6

3. Kavousi M, Elias-Smale S, Rutten JH, Leening MJ, 
Vliegenthart R, Verwoert GC, et al. Evaluation of newer risk markers for coronary heart disease risk classification: $\mathrm{A}$ cohort study. Ann Intern Med. 2012;156(6):438-44. https://doi. org/10.7326/0003-4819-156-6-201203200-00006 PMid:22431676

4. Yeboah J, Young R, McClelland RL, Delaney JC, Polonsky TS, Dawood FZ, et al. Utility of nontraditional risk markers in atherosclerotic cardiovascular disease risk assessment. J Am Coll Cardiol. 2016;67(2):139-47.

PMid:26791059

5. Libby $P$, Ridker PM, Hansson GK. Inflammation in atherosclerosis: From pathophysiology to practice. J Am Coll Cardiol. 2009;54(23):2129-38.

PMid:19942084

6. Graham I, Atar D, Borch-Johnsen K, Boysen G, Burell G, Cifkova $\mathrm{R}$, et al. European guidelines on cardiovascular disease prevention in clinical practice: Full text. Fourth joint task force of the European society of cardiology and other societies on cardiovascular disease prevention in clinical practice (constituted by representatives of nine societies and by invited experts). Eur J Cardiovasc Prev Rehabil. 2007;14(2):S1-113. https://doi.org/10.3410/f.718277733.793490996

PMid: 17726407

7. Kuller LH, Tracy RP, Shaten J, Meilahn EN. Relation of $\mathrm{C}$-reactive protein and coronary heart disease in the MRFIT nested case-control study. Multiple risk factor intervention trial. Am J Epidemiol. 1996;144(6):537-47. https://doi.org/10.1093/ oxfordjournals.aje.a008963

PMid:8797513

8. Ridker PM, Rifai N, Rose L, Buring JE, Cook NR. Comparison of C-reactive protein and low-density lipoprotein cholesterol levels in the prediction of first cardiovascular events. N Engl J Med. 2002;347(20):1557-65. https://doi.org/10.1056/nejmoa021993 PMid:12432042

9. Kaptoge S, DiAngelantonio E, Lowe G, Pepys MB, Thompson SG, Collins $\mathrm{R}$, et al. C-reactive protein concentration and risk of coronary heart disease, stroke and mortality: An individual participant meta-analysis. Lancet. 2010;375(9790):132-40. https://doi.org/10.1016/s0140-6736(09)61717-7 PMid:20031199

10. Ridker PM, Danielson E, Fonseca FA, Genest J, Gotto AM Jr., Kastelein $\mathrm{JJ}$, et al. Rosuvastatin to prevent vascular events in men and women with elevated C-reactive protein. N Engl J Med. 2008;359(21):2195-207. https://doi.org/10.1016/j. jvs.2008.12.037 PMid:18997196

11. Kaul S, Morrissey RP, Diamond GA. By Jove? What is a clinician to make of JUPITER? Arch Intern Med. 2010;170(12):1073-77. https://doi.org/10.1001/archinternmed.2010.189 PMid:20585074

12. Lubrano V, Balzan S. Consolidated and emerging inflammatory markers in coronary artery disease. World J Exp Med. 2015;5(1):21-32.

PMid:25699231

13. Lubrano V, Balzan S. Enzymatic antioxidant system in vascular inflammation and coronary artery disease. World J Exp Med. 2015;5(4):218-24. https://doi.org/10.5493/wjem.v5.i4.218 PMid:26618108

14. Subirana I, Montserrat F, Diaz O, Vila J, Francés A, Delpon E, et al. Prediction of coronary disease incidence by biomarkers of inflammation, oxidation, and metabolism. Sci Rep. 2018;8:3191.

15. Karu I, Starkopf J, Zilmer K, Zilmer M. Growth factors serum levels in coronary artery disease patients scheduled for bypass surgery: Perioperative dynamics and comparisons with healthy volunteers. Biomed Res Int. 2013;2013:985404. https://doi. org/10.1155/2013/985404

PMid:23984427

16. Kleveland O, Kunszt G, Bratlie M, Ueland T, Broch K, Holte E, et al. Effect of a Single Dose of the Interleukin-6 Receptor Antagonist on Inflammation and Troponin Release in Patients with Non-STEMI. Tamil Nadu: ESC; 2015.

17. Ridker PM. From C-reactive protein to interleukin-6 to interleukin-1: Moving upstream to identify novel targets for atheroprotection. Circ Res. 2016;118(1):145-56. https://doi. org/10.1161/circresaha.115.306656

PMid:26837745

18. Mach F, Baigent $C$, Catapano AL, Koskinas KC, Casula M, Badimon L, et al. $2019 \mathrm{ESC} / \mathrm{EAS}$ guidelines for the management of dyslipidaemias: Lipid modification to reduce cardiovascular risk. Eur Heart J. 2019;41(1):111-88. https://doi.org/10.341 O/f.718277728.793490990

PMid:31504418

19. Emerging Risk Factors Collaboration. Diabetes mellitus, fasting blood glucose concentration, and risk of vascular disease: A collaborative meta-analysis of 102 prospective studies. Lancet. 2010;375(9733):2215-22. https://doi.org/10.1016/ s0140-6736(10)60484-9

PMid:20609967

20. Odegaard AO, Jacobs DR Jr., Sanchez OA, Goff DC Jr., Reiner AP, Gross MD. Oxidative stress, inflammation, endothelial dysfunction and incidence of Type 2 diabetes. Cardiovasc Diabetol. 2016;15:51. https://doi.org/10.1186/ s12933-016-0369-6

PMid:27013319

21. Shu L, Chan KH, Zhang G, Huan T, Kurt Z, Zhao Y, et al. Shared genetic regulatory networks for cardiovascular disease and Type 2 diabetes in multiple populations of diverse ethnicities in the United States. PLoS Genet. 2017;13(9):e1007040. https:// doi.org/10.1371/journal.pgen.1007040

PMid:28957322

22. Ridker PM, Bhatt DL, Bavry AA. Justification for the Use of Statins in Prevention: An Intervention Trial Evaluating RosuvastatinJUPITER. United States: American Heart Association Annual Scientific Sessions; 2015.

23. Jing $Y$, Zhu $D, B i ~ Y$, Yang $D, H u ~ Y$, Shen S. Monocyte chemoattractant protein 1-2518 A/G polymorphism and susceptibility to Type 2 diabetes in a Chinese population. Clin Chim Acta. 2011;412(5-6):466-9. https://doi.org/10.1016/j. cca.2010.11.030

24. Khera A, McGuire DK, Murphy SA, Stanek HG, Das SR, Vongpatanasin $\mathrm{W}$, et al. Race and gender differences in C-reactive protein levels. J Am Coll Cardiol. 2005;46(3):464-9. PMid:16053959

25. Milan-Mattos JC, Anibal FF, Perseguini NM, Minatel V, RehderSantos P, Castro CA, et al. Effects of natural aging and gender on pro-inflammatory markers. Braz $\mathrm{J}$ Med Biol Res. 2019;52(9):e8392. https://doi.org/10.1590/1414-431x20198392 PMid:31411315

26. Kumari R, Kumar S, Ahmad MK, Singh R, Pradhan A Chandra $S$, et al. TNF- $\alpha /$ IL-10 ratio: An independent predictor for coronary artery disease in North Indian population. Diabetes Metab Syndr. 2018;12(3):221-5. https://doi.org/10.1016/j. dsx.2017.09.006

PMid:28988596

27. Tanaka T, Narazaki M, Kishimoto T. IL-6 in inflammation, immunity and disease. Cold Spring Harb Perspect Biol. 2014;6(10):a016295. https://doi.org/10.1101/cshperspect. a016295 PMid:25190079

28. Huang YH, Yang HY, Huang SW, Ou G, Hsu YF, Hsu MJ. 
Interleukin-6 induces vascular endothelial growth factor-C expression via src-FAK-STAT3 signaling in lymphatic endothelial cells. PLoS One. 2016;11(7):e0158839. https://doi.org/10.1371/ journal.pone. 0158839

PMid:27383632

29. Zepeda-Orozco D, Wen HM, Hamilton BA, Raikwar NS, Thomas CP. EGF regulation of proximal tubule cell proliferation and VEGF-A secretion. Physiol Rep. 2017;5(18):e13453. https://doi.org/10.14814/phy2.13453

PMid:28963126

30. Rosenson RS, Brewer HB, Ansell B, Barter P, Chapman MJ, Heinecke JW, et al. Translation of high-density lipoprotein function into clinical practice: Current prospects and future challenges. Circulation. 2013;128(11):1256-67. https://doi. org/10.1161/circulationaha.113.000962 PMid:24019446

31. Asztalos BF, Tani M, Schaefer EJ. Metabolic and functional relevance of HDL subspecies. Curr Opin Lipidol. 2011;22(3):17685. https://doi.org/10.1097/mol.0b013e3283468061 PMid:21537175

32. White CR, Datta G, Mochon P, Zhang Z, Kelly O, Curcio C, et al. Vasculoprotective effects of apolipoprotein mimetic peptides: An evolving paradigm in HDL therapy (vascular disease prevention, In Press). Vasc Dis Prev. 2009;6:122-30. https://doi. org/10.2174/1567270000906010122

PMid:20084185

33. Wojdasiewicz P, Poniatowski LA, Szukiewicz D. The role of inflammatory and anti-inflammatory cytokines in the pathogenesis of osteoarthritis. Mediators Inflamm. 2014;2014:561459. https:// doi.org/10.1155/2014/561459

PMid:24876674

34. Woodward EA, Prêle CM, Nicholson CE, Kolesnik TB, Hart PH. The anti-inflammatory effects of interleukin-4 are not mediated by suppressor of cytokine signalling-1 (SOCS1). Immunology. 2010;131(1):118-27. https://doi. org/10.1111/j.1365-2567.2010.03281.x PMid:20406299

35. Lee YW, Kim PH, Lee WH, Hirani AA. Interleukin-4, oxidative stress, vascular inflammation and atherosclerosis. Biomol Ther (Seoul). 2010;18(2):135-44. PMid:21072258

36. Orsó E, Schmitz G. Lipoprotein(a) and its role in inflammation, atherosclerosis and malignancies. Clin Res Cardiol Suppl. 2017;12(1):31-7. https://doi.org/10.1007/s11789-017-0084-1

\section{PMid:28188431}

37. Lyngdoh T, Marques-Vidal P, Paccaud F, Preisig M, Waeber G Bochud $\mathrm{M}$, et al. Elevated serum uric acid is associated with high circulating inflammatory cytokines in the populationbased Colaus study. PLoS One. 2011;6(5):e19901. https://doi. org/10.1371/journal.pone.0019901

PMid:21625475

38. Panee J. Monocyte chemoattractant protein 1 (MCP-1) in obesity and diabetes. Cytokine. 2012;60(1):1-12. https://doi. org/10.1016/j.cyto.2012.06.018

PMid:22766373

39. Ferrante $\mathrm{G}$, Condorelli $\mathrm{G}$. Interleukin-6 trans-signaling and risk of future cardiovascular events: A new avenue of atheroprotection? Cardiovasc Res. 2019;115(1):8-9. https://doi.org/10.1093/cvr/ cvy233 PMid:30219834

40. Ridker PM. From C-reactive protein to interleukin-6 to interleukin-1: Moving upstream to identify novel targets for atheroprotection. Circ Res. 2016;118(1):145-56. https://doi. org/10.1161/circresaha.115.306656

PMid:26837745

41. Wannamethee SG, Whincup PH, Rumley A, Lowe GD Inter-relationships of interleukin-6, cardiovascular risk factors and the metabolic syndrome among older men. J Thromb Haemost. 2007;5(8):1637-43. https://doi. org/10.1111/j.1538-7836.2007.02643.x

PMid:17596140

42. Bacchiega $B C$, Bacchiega $A B$, Usnayo $M J$, Bedirian $R$, Singh G, da Rocha Castelar Pinheiro G. Interleukin-6 inhibition and coronary artery disease in a high-risk population: A prospective community-based clinical study. J Am Heart Assoc. 2017;6(3):e005038. https://doi.org/10.1161/jaha.116.005038 PMid:28288972

43. Lin $\mathrm{YH}$, Glei $\mathrm{D}$, Weinstein $\mathrm{M}, \mathrm{Wu} \mathrm{SI}$, Chien $\mathrm{KL}$. Additive value of interleukin- 6 and C-reactive protein in risk prediction for allcause and cardiovascular mortality among a representative adult cohort in Taiwan. J Form Med Assoc. 2017;116(12):98292. https://doi.org/10.1016/j.jma.2017.02.002

44. Garofallo SB, Portal VL, Markoski MM, Dias LD, de Quadrosa AS, Marcadenti A. Correlations between traditional and nontraditional indicators of adiposity, inflammation, and monocyte subtypes in patients with stable coronary artery disease. J Obes. 2019;2019:3139278. https://doi.org/10.1155/2019/3139278 PMid:31354986 


\section{Appendices}

\section{Appendix 1}

Glycemic profile - Glucose concentration was determined by a hexokinase enzymatic method (NADPH production is measured spectrophotometrically at $340 \mathrm{~nm}$ using COBAS INTEGRA Glucose HK Gen. 3). HgA1c concentration was determined by a turbidimetric inhibition-based immunoassay (COBAS INTEGRA, Tina-quant Gen.2, Roche Diagnostics).

Lipoprotein profile-Cholesterol concentration was determined using a colorimetric enzymatic method (color intensity is measured spectrophotometrically at $512 \mathrm{~nm}$ using COBAS INTEGRA Cholesterol Gen.2 (CHOL2), Test CHOL2, Test ID 0-586). HDL-C concentration was determined by a homogenous colorimetric enzymatic method (Color intensity is measured spectrophotometrically at $583 \mathrm{~nm}$ using COBAS INTEGRA HDL-cholesterol plus $2^{\text {nd }}$ generation (HDL-C); HDL-C test, ID 0-201). LDL-C was determined by a homogenous colorimetric enzymatic method (color intensity is measured spectrophotometrically at $583 \mathrm{~nm}$ using COBAS INTEGRA LDL-cholesterol plus $2^{\text {nd }}$ generation (LDL-C); LDL-C Test, ID 0-301). Triglycerides were determined by a colorimetric enzymatic method (color intensity is measured spectrophotometrically at $512 \mathrm{~nm}$ using COBAS INTEGRA triglycerides (TRIGL); test TRIGL, test ID 0-010). ApoAI and ApoB were measured turbidimetrically at $340 \mathrm{~nm}$, using anti-ApoAI and anti-ApoB antiserum (COBAS INTEGRA Tina-quant Apolipoprotein A-1ver.2 and COBAS INTEGRA Tinaquant Apolipoprotein B ver.2, Roche). Lp(a) was determined turbidimetrically at 800/660 nm (COBAS INTEGRA Tina-quant ${ }^{\circledR}$ Lipoprotein (a) Gen. 2, Roche).

Inflammatory profile - Quantitative determination of IL-1A, IL-1B, IL-2, IL-4, IL-6, IL-8, IL-10, TNF- $\alpha$, INF$\gamma$, MCP-1, EGF, and VEGF was done using a commercial Randox cytokine and growth factors array kit, based on a sandwich chemiluminescent immune test. The concentration of C-reactive protein was determined by a latex immunoturbidimetric method (COBAS INTEGRA Tina-quant ${ }^{\circledR}$ CRP (Latex) assay, Roche). 